Original article

\title{
Synthesis of tacrine-lophine hybrids via one-pot four component reaction and biological evaluation as acetyl- and butyrylcholinesterase inhibitors
}

\author{
Jessé Sobieski da Costa ${ }^{a}$, João Paulo Bizarro Lopes ${ }^{a}$, Dennis Russowsky ${ }^{a}$, \\ Cesar Liberato Petzhold ${ }^{a}$, Antonio César de Amorim Borges ${ }^{a}$, Marco Antonio Ceschi ${ }^{\mathrm{a}, *}$, \\ Eduardo Konrath $^{\mathrm{b}}$, Cristiane Batassini ${ }^{\mathrm{b}}$, Paula Santana Lunardi ${ }^{\mathrm{b}}$, \\ Carlos Alberto Saraiva Gonçalves ${ }^{\mathrm{b}}$
}

${ }^{a}$ Instituto de Química, Universidade Federal do Rio Grande do Sul, Av. Bento Gonçalves, 9500, Campus do Vale, 91501-970 Porto Alegre - RS, Brazil

${ }^{\mathrm{b}}$ Departamento de Bioquímica, Universidade Federal do Rio Grande do Sul, Rua Ramiro Barcelos, 2600, Prédio Anexo, 90035-003, Brazil

\section{A R T I C L E I N F O}

\section{Article history:}

Received 12 September 2012

Received in revised form

23 January 2013

Accepted 24 January 2013

Available online 4 February 2013

\section{Keywords:}

Tacrine

Lophine

Four component reaction

Indium trichloride

AChE inhibitory activity

\begin{abstract}
A B S T R A C T
A novel series of tacrine-lophine hybrids was synthesized and tested for their ability to inhibit acetylcholinesterase (AChE) and butyrylcholinesterase (BuChE) with $\mathrm{IC}_{50}$ in the nanomolar concentration scale. The key step is the one-pot four component condensation reaction of 9-aminoalkylamino-1,2,3,4tetrahydroacridines, benzil, different substituted aromatic aldehydes and $\mathrm{NH}_{4} \mathrm{OAc}$, using $\mathrm{InCl}_{3}$ as the best catalyst. Tacrine-lophine hybrids were found to be potent and selective inhibitors of cholinesterases. As an extension of the four component approach to tetrasubstituted imidazoles, a new series of bis-(2,4,5triphenyl-1H-imidazoles) or bis(n)-lophines was tested against AChE and BuChE.
\end{abstract}

(c) 2013 Elsevier Masson SAS. All rights reserved.

\section{Introduction}

Alzheimer's disease (AD) is a progressive neurodegenerative disorder of the central nervous system (CNS), characterized by deposits of aberrant proteins namely $\beta$-amyloid $(A \beta)$ and $\tau$-protein, oxidative stress, loss of synapses and death of cholinergic neurons [1]. The etiopathogenesis of AD still remains unknown although, in the last decades, several involved factors have been identified and found consistent with its onset. Among them is the impairment of the cholinergic system, as indicated by the presence of altered cholinergic markers in $\mathrm{AD}$ patients, resulting in a pronounced acetylcholine $(\mathrm{ACh})$ deficiency which translates into a generalized withdrawal of cholinergic tone [2-4]. Nonetheless, interest in developing improved $\mathrm{ChE}$ inhibitors for treatment of $\mathrm{AD}$ has increased recently, likely due to the body of empirical evidence showing the benefits of $\mathrm{ChE}$ inhibitors in $\mathrm{AD}$ patients as well as the lack of successful alternative approaches [5-7].

\footnotetext{
* Corresponding author. Tel.: +55 51 33087205; fax: +55 5133087304 .

E-mail address: mceschi@iq.ufrgs.br (M.A. Ceschi).
}

Acetylcholinesterase (AChE) and butyrylcholinesterase (BuChE) are enzymes which constitute the group of the cholinesterases. Acetylcholinesterase hydrolyses acetylcholine and is mainly associated with nerves and muscles, being typically found on the synapses, while butyrylcholinesterase hydrolyses butyrylcholine and is synthesized by the liver, being found in large concentration in serum [8].

The current therapeutic options for the treatment of AD are limited to four AChE inhibitors [9,10], namely donepezil, rivastigmine, galantamine and tacrine, and one $\mathrm{N}$-methyl-D-aspartate receptor antagonist, memantine [11]. These compounds have been approved by the U.S. Food and Drug Administration for the treatment of AD.

In healthy brains, AChE hydrolyzes the majority of acetylcholine while BuChE plays a secondary role. However, as AD progresses, the activity of AChE decreases, while that of BuChE significantly increases in the hippocampus and temporal cortex [12].

All current kinetic models for AChE propose the existence of at least two substrate-binding sites; the active site and the peripheral anionic site (PAS). Inhibitors directed to the active site prevent the binding of the acetylcholine, or its hydrolysis, by occupying the site with a high affinity molecule, such as tacrine. The active site is composed of two subsites. In the catalytic anionic subsite (CAS), it 
has been proposed that the choline moiety in AChE is stabilized principally via a cation- $\pi$ interaction with $\operatorname{Trp} 84$, and also interacts with Glu199 and Phe330 [5,13]. A similar cation- $\pi$ interaction occurs in human BuChE, where Trp82 interacts with the product choline and the substrate butyrylthiocholine [14].

Tacrine, the first approved drug for $\mathrm{AD}$, is a potent nonselective inhibitor of both AChE and BuChE. Although this lack of selectivity, and hepatotoxicity, has reduced its therapeutic use, it has been the most studied compound and remains a reference structure in the development of new ChEs inhibitors as potential drugs for AD.

About fifteen years ago, bis(n)-tacrine analogues linked by an alkylene chain were prepared, and it was proved that these dimeric molecules of tacrine offered a much stronger potency and selectivity toward AChE [15]. Bis(7)-tacrine simultaneously binds at both the CAS and the PAS sites and provides a higher selectivity towards AChE over BuChE. Since then, most of the dual binding site AChE inhibitors developed so far contain at least one unit related to tacrine, probably because of its ease of synthesis and its affinity for both the CAS and PAS sites of AChE, and an enlarged biological profile [16].

Among achievements in synthesis and pharmacomodulation, various tacrine-based heterodimers have been designed and studied. These tacrine heterodimers are usually obtained by connecting natural or synthetic compounds by a linker of suitable length. Examples of tacrine-based heterodimer families include the combination of tacrine with structural units such as tacrine-huperzine A [17-19], tacrine-donepezil [16,20,21], tacrine-indole [22], tacrine4-Oxo-4H-chromene [10] and tacrine-8-hydroxyquinoline [23].

In continuation with our search for new $\mathrm{ChE}$ inhibitors based on tacrine units $[24,25]$ as well as the development of new synthetic methods using Lewis acids as catalysts [25-27], we describe in the present study, the synthesis of a novel class of hybrids tacrine2,4,5-triphenyl- $1 \mathrm{H}$-imidazole (or tacrine-lophine hybrids) as inhibitors of AChE and BuChE (Scheme 1). Compounds containing imidazole moiety have many pharmacological properties and play important roles in biochemical processes [28,29]. As an extension of the four component approach to tetrasubstituted imidazoles, we also synthesized a new series of bis-(2,4,5-triphenyl- $1 \mathrm{H}$-imidazoles) or bis(n)-lophines (Scheme 2). These non-based tacrine dimers were also subjected to inhibitory essays to AChE and BuChE.
The key step is a one-pot four component synthesis of 1,2,4,5tetrasubstituted imidazoles catalyzed by Lewis acid.

\section{Results and discussion}

\subsection{Chemistry}

The general synthesis for the novel hybrids tacrine-2,4,5triphenylimidazole $\mathbf{2} \mathbf{a}-\mathbf{g}, \mathbf{3 a}-\mathbf{g}, \mathbf{4 a}-\mathbf{g}, \mathbf{5}$ and $\mathbf{6}$ is depicted in Scheme 1 . The key step is the one-pot four component condensation reaction of 9-aminoalkylamino-1,2,3,4-tetrahydroacridines, benzil, different substituted aromatic aldehydes and $\mathrm{NH}_{4} \mathrm{OAc}$ using Lewis acid as catalyst. According to a previously reported protocol, 9chloro-1,2,3,4-tetrahydroacridine was first prepared by the cyclization of anthranilic acid with cyclohexanone [30,31]. In order to introduce the side chain, different alkylenediamines reacted with 9chloro-1,2,3,4-tetrahydroacridine to generate the 9-aminoalkylamino-1,2,3,4-tetrahydroacridines in good yields (87-95\%) [32].

Despite the use of Lewis acid catalyst on the multicomponent synthesis of 1,2,4,5-tetrasubstituted imidazoles, Sharma and coworkers found $\mathrm{InCl}_{3} \cdot 3 \mathrm{H}_{2} \mathrm{O}$ as a mild and effective catalyst [29]. Thus, in order to evaluate the scope of the multicomponent synthesis of hybrids tacrine-2,4,5-triphenyl- $1 \mathrm{H}$-imidazole, we chose compound 1c as the substrate, the aim being to examine some of the general features of these reactions, such as Lewis acid catalysts, stoichiometry, solvent, temperature and product conversion.

As shown in Table 1 (entry 1), we found that the use of $\mathrm{InCl}_{3}$ (15 mol\%) under ethanol reflux for $96 \mathrm{~h}$, afforded 2a in quite good yield (55\%). This developed protocol was applied to the synthesis of tacrine-2,4,5-triphenyl- $1 H$-imidazole $2 \mathbf{a}-\mathbf{g}, 3 \mathbf{a}-\mathbf{g}, \mathbf{4 a}-\mathbf{g}, \mathbf{5}$ and $\mathbf{6}$ which have a lophine framework substituted at the meta- or paraposition of the 2-phenyl imidazole ring, as shown in Table 2. Next, bis(n)-lophines $\mathbf{7 a}-\mathbf{e}$ were synthesized by using a similar protocol as depicted in Scheme 2.

\subsection{AChE and BuChE inhibition evaluation}

The inhibitory activities against $\mathrm{AChE}$ and BuChE of new tacrinelophine hybrids and bis(n)lophines, together with that of bis(7)-<smiles>CC(C)Nc1c2c(nc3ccccc13)CCCC2</smiles>

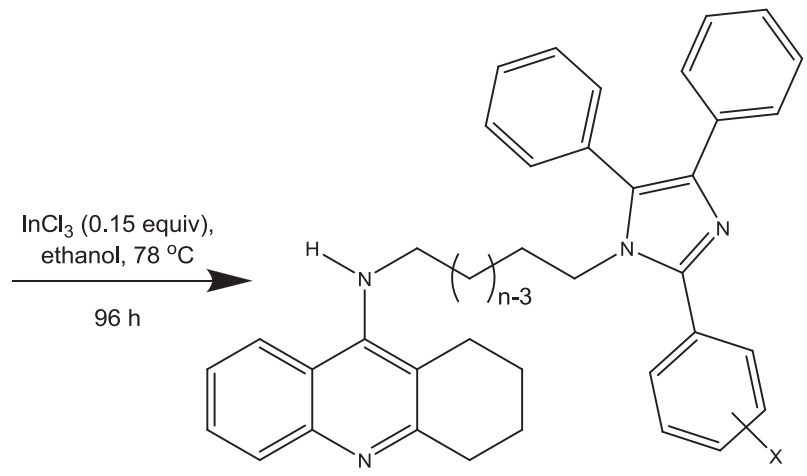

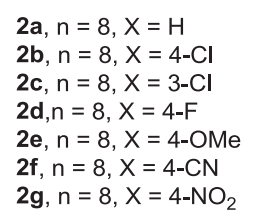

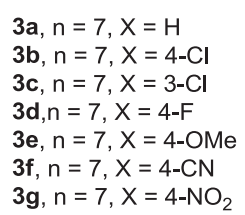
$3 c, n=7, x=3-C l$ $n=7, X=4-F$

3f, $n=7, X=4-\mathrm{CN}$ $3 \mathrm{~g}, \mathrm{n}=7, \mathrm{X}=4-\mathrm{NO}_{2}$

$$
\begin{aligned}
& \mathbf{4 a}, n=6, X=H \\
& \mathbf{4 b}, n=6, X=4-\mathrm{Cl} \\
& \mathbf{4 c}, n=6, X=3-\mathrm{Cl} \\
& \mathbf{4 d}, n=6, X=4-\mathrm{F} \\
& \mathbf{4 e}, n=6, X=4-\mathrm{OMe} \\
& \mathbf{4 f}, \mathrm{n}=6, X=4-\mathrm{CN} \\
& \mathbf{4 g}, \mathrm{n}=6, X=4-\mathrm{NO}_{2} \\
& \mathbf{5}, \mathrm{n}=9, X=4-\mathrm{Cl} \\
& \mathbf{6}, \mathrm{n}=10, X=4-\mathrm{Cl}
\end{aligned}
$$

Scheme 1. Synthesis of tacrine-lophine hybrids. 


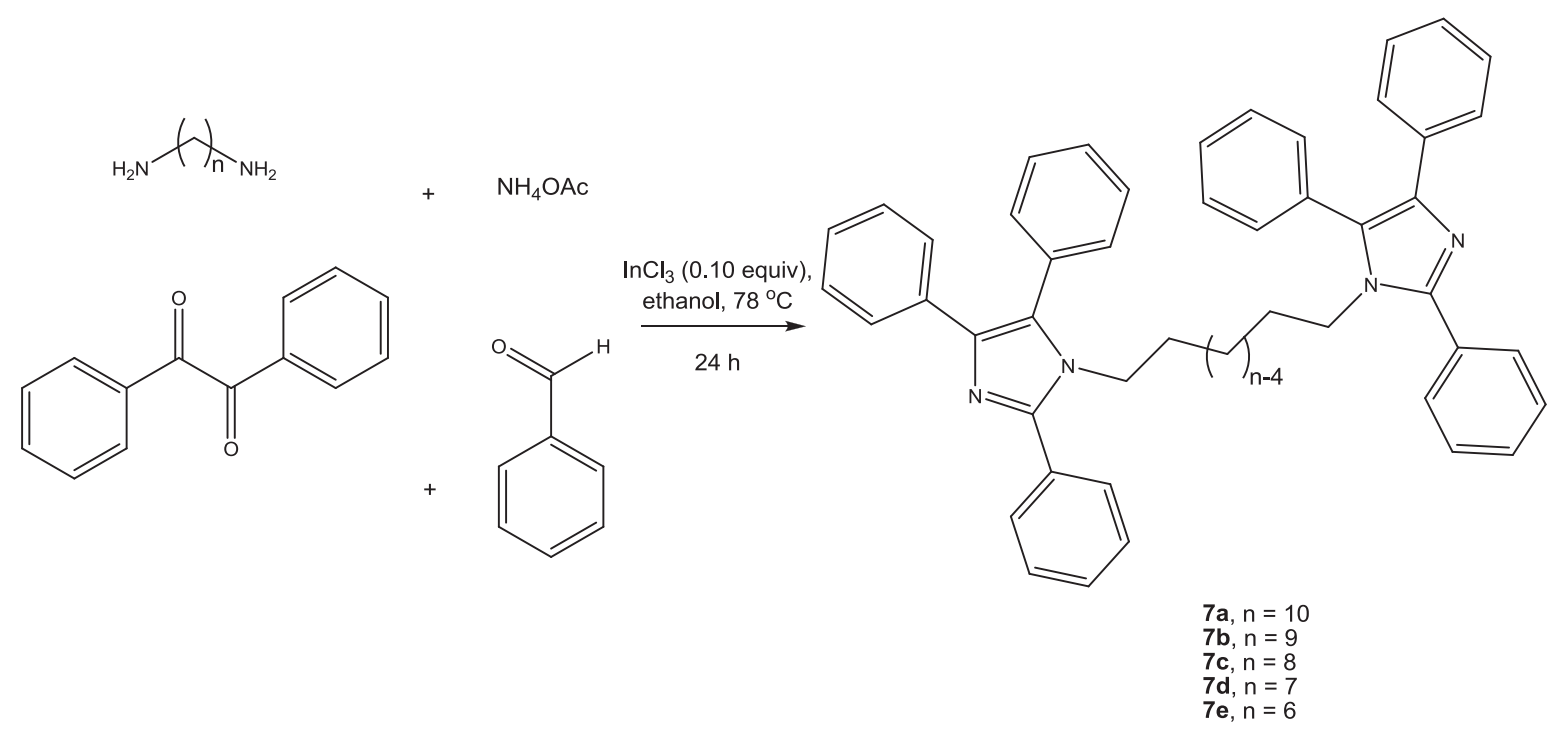

Scheme 2. Synthesis of bis(n)-lophines.

tacrine taken as reference, are reported in Table 2, and are expressed as $\mathrm{IC}_{50}$ values. Tacrine-lophine hybrids were found to be potent inhibitors of cholinesterases with $\mathrm{IC}_{50}$ in the nanomolar concentration scale. The most active AChE inhibitor in the assayed series of tacrine-lophines was hybrid $\mathbf{2} \mathbf{b}(X=4-\mathrm{Cl})$ with its $\mathrm{IC}_{50}$ of $5.87 \mathrm{nM}$ (entry 2 ), bearing an octane chain between the tacrine and lophine moieties, pointing out that this linker allows optimal interaction between the aromatic fragments of such an inhibitor and the CAS and PAS of AChE. Moreover, compound $\mathbf{2 b}$ showed very high selectivity for AChE over BuChE (18.5-fold), comparing to only 2.2-fold BuChE selectivity seen with bis(7)-tacrine (entry 29). From this dataset with octamethylene linker, compounds $2 \mathbf{d}(X=4-\mathrm{F})$ and $2 \mathbf{e}(X=4-\mathrm{OMe})$ were active for both $\mathrm{AChE}$ and BuChE, compounds $2 \mathrm{f}(X=4-\mathrm{CN})$ and $2 \mathrm{~g}\left(X=4-\mathrm{NO}_{2}\right)$ were active only for BuChE while $\mathbf{2 a}(X=\mathrm{H})$ and $\mathbf{2 c}(X=3-\mathrm{Cl})$ were inactive toward both enzymes.

In the dataset of tacrine-lophines with the heptane chain, compound $3 \mathbf{f}(R=4-\mathrm{CN})$ entry 13 , showed the best inhibition for BuChE in the assayed series with $\mathrm{IC}_{50}$ of $7.10 \mathrm{nM}$ and was inactive toward AChE. It is noteworthy that $\mathbf{3 f}$ was an effective inhibitor of BuChE and exhibited more potent inhibition value compared with prototype bis(7)-tacrine. With the same chain length, compounds 3a $(\mathrm{R}=\mathrm{H})$, 3b $(R=4-\mathrm{Cl})$, 3d $(R=4-\mathrm{F})$, 3e $(R=4-\mathrm{OMe}), 3 \mathbf{g}(R=4$ $\left.\mathrm{NO}_{2}\right)$ were active for both $\mathrm{AChE}$ and BuChE, while $3 \mathbf{c}(X=3-\mathrm{Cl})$ was the only compound inactive for both enzymes in this dataset. In the case of hexane chain, none of the compounds $\mathbf{4 a}-\mathbf{4 g}$ exhibited AChE activity while compounds $\mathbf{4 a}-\mathbf{4 g}$ exhibited BuChE inhibitory $\mathrm{IC}_{50}$ values ranging from 12.44 to $58.00 \mathrm{nM}$. Comparing products possessing the same substituents in the 2-phenyl group fragment, it was possible to detect the effective influence of changes in the substructure of lophine on the inhibition of ChEs. In the case of chloride substituent in the para-position of 2-phenyl group (entries 2, 9 and 16) when the length of the linker is made smaller from the optimum octane chain (entry 2), the ability to inhibit AChE progressively diminished. On the other hand, for compounds containing the chloride substituent in the para-position (entries 2, 9 and 16), an increased inhibitory activity against BuChE was observed. Also, hybrids with chloride substituent in the meta-position (entries 3 and 10) were inactive toward AChE. This result could be explained as a consequence of the fact that all phenyl rings bonded to the imidazole core are not coplanar and the dihedral angles between the phenyl ring planes can be increased with substituent groups in the meta-position [33]. So, it can be inferred that the loss of AChE activities with substituent at phenyl metasubstituted hybrids results from a smaller $\pi-\pi$ interaction of lophine framework with the active sites of the enzyme. On the other hand, this can explain the improved AChE inhibitor $2 \mathbf{b}$ bearing a phenyl para-substituted which has smaller dihedral angles on the phenyl ring planes of the lophine framework, favoring binding interactions.

On the bis(n)-lophines dataset, homodimer 7c (entry 26) proved to be an interesting compound as a non-tacrine dimer, showing selective AChE inhibitory activity with its $\mathrm{IC}_{50}$ of $42.55 \mathrm{nM}$.

\section{Conclusion}

In summary, we have developed a simple and convenient approach for the synthesis of tacrine-lophine and lophine-lophine dimers via one-pot four component condensation reaction using $\mathrm{InCl}_{3}$ as catalyst. Several tacrine-lophine hybrids were found to be potent and selective inhibitors of $\mathrm{AChE}$ and BuChE with $\mathrm{IC}_{50}$ in the nanomolar concentration scale. The non-tacrine bis(8)-lophine (7c) showed a selective AChE inhibitory activity with its $\mathrm{IC}_{50}$ value of

Table 1

One-pot four component condensation reaction for the synthesis of hybrid 2a under different conditions.

\begin{tabular}{lllccc}
\hline Entry & $\begin{array}{l}\text { Catalyst } \\
(15 \mathrm{~mol} \%)\end{array}$ & Solvent & $\begin{array}{c}\text { Temp. } \\
\left({ }^{\circ} \mathrm{C}\right)\end{array}$ & $\begin{array}{l}\text { Reaction } \\
\text { time }(\mathrm{h})\end{array}$ & Yield (\%),b \\
\hline 1 & $\mathrm{InCl}_{3}$ & Ethanol & 78 & 96 & $55^{\mathrm{c}}$ \\
2 & $\mathrm{FeCl}_{3}$ & Ethanol & 78 & 96 & $45^{\mathrm{c}}$ \\
3 & $\mathrm{SnCl}_{2} \cdot 2 \mathrm{H}_{2} \mathrm{O}$ & Ethanol & 78 & 96 & $41^{\mathrm{c}}$ \\
4 & $\mathrm{AlCl}_{3}$ & Ethanol & 78 & 96 & $44^{\mathrm{c}}$ \\
5 & $\mathrm{BF}_{3} \cdot \mathrm{Et}_{2} \mathrm{O}$ & Ethanol & 78 & 96 & $33^{\mathrm{c}}$ \\
6 & $\mathrm{InCl}_{3}$ & Free solvent & 120 & 19 & 3 \\
7 & $\mathrm{InCl}_{3}$ & n-Pentanol & 120 & 48 & $11^{\mathrm{d}}$ \\
8 & $\mathrm{InCl}_{3}$ & Ethanol & 25 & 180 & 12 \\
9 & $\mathrm{InCl}_{3}$ & Ethanol & 78 & 48 & $31^{\mathrm{d}}$ \\
10 & $\mathrm{InCl}_{3}$ & Ethanol & 78 & 96 & 35 \\
\hline
\end{tabular}

${ }^{a}$ All yields refer to purified product $\mathbf{2 a}$

b The reactions were performed using 1.0 equivalent of 9-octylamino-1,2,3,4tetrahydroacridine 1c, benzaldehyde, benzil and ammonium acetate.

c After $48 \mathrm{~h}$, one more equivalent of benzaldehyde, benzil and ammonium acetate was added.

d After $24 \mathrm{~h}$, one more equivalent of benzaldehyde, benzil and ammonium acetate was added. 
Table 2

Inhibitory activity on $\mathrm{AChE}$ and $\mathrm{BuChE}$, and $\mathrm{IC}_{50}$ ratio of the studied compounds.

\begin{tabular}{|c|c|c|c|c|c|c|c|}
\hline \multirow[t]{2}{*}{ Entry } & \multirow[t]{2}{*}{ Compd } & \multirow[t]{2}{*}{$n$} & \multirow[t]{2}{*}{$X$} & \multirow{2}{*}{$\begin{array}{l}\text { Yield } \\
(\%)^{\mathrm{a}}\end{array}$} & \multicolumn{2}{|l|}{$\mathrm{IC}_{50}(\mathrm{nM})^{\mathrm{b}}$} & \multirow{2}{*}{$\begin{array}{l}\mathrm{IC}_{50} \text { ratio } \\
\text { BuChE/AChE }\end{array}$} \\
\hline & & & & & AChE & BuChE & \\
\hline 1 & $2 a$ & 8 & $\mathrm{H}$ & 55 & n.a. & n.a. & - \\
\hline 2 & $2 \mathbf{b}$ & 8 & $4-\mathrm{Cl}$ & 57 & $5.87 \pm 0.30$ & $108.97 \pm 6.41$ & 18.5 \\
\hline 3 & $2 c$ & 8 & $3-\mathrm{Cl}$ & 37 & n.a. & n.a. & - \\
\hline 4 & 2d & 8 & $4-\mathrm{F}$ & 41 & $25.78 \pm 4.66$ & $57.30 \pm 4.15$ & 2.2 \\
\hline 5 & $2 e$ & 8 & 4-OMe & 74 & $125.90 \pm 15.84$ & $82.34 \pm 4.51$ & 0.6 \\
\hline 6 & $2 f$ & 8 & $4-\mathrm{CN}$ & 33 & n.a. & $30.47 \pm 3.65$ & - \\
\hline 7 & $2 \mathrm{~g}$ & 8 & $4-\mathrm{NO}_{2}$ & 39 & n.a. & $120.13 \pm 29.25$ & - \\
\hline 8 & $3 a$ & 7 & $\mathrm{H}$ & 54 & $80.65 \pm 4.90$ & $92.42 \pm 1.95$ & 1.1 \\
\hline 9 & 3b & 7 & $4-\mathrm{Cl}$ & 33 & $59.76 \pm 2.09$ & $37.57 \pm 6.24$ & 0.6 \\
\hline 10 & $3 c$ & 7 & $3-\mathrm{Cl}$ & 42 & n.a. & n.a. & - \\
\hline 11 & 3d & 7 & $4-\mathrm{F}$ & 52 & $38.51 \pm 3.63$ & $67.31 \pm 4.79$ & 1.7 \\
\hline 12 & $3 e$ & 7 & 4-OMe & 44 & $68.74 \pm 7.10$ & $61.69 \pm 6.71$ & 0.9 \\
\hline 13 & 3f & 7 & $4-\mathrm{CN}$ & 53 & n.a. & $7.10 \pm 2.49$ & - \\
\hline 14 & $3 g$ & 7 & $4-\mathrm{NO}_{2}$ & 44 & $72.10 \pm 2.18$ & $76.89 \pm 28.13$ & 1.0 \\
\hline 15 & $4 a$ & 6 & $\mathrm{H}$ & 53 & n.a. & $58.00 \pm 5.37$ & - \\
\hline 16 & $4 b$ & 6 & $4-\mathrm{Cl}$ & 37 & n.a. & $29.28 \pm 2.67$ & - \\
\hline 17 & $4 c$ & 6 & $3-\mathrm{Cl}$ & 29 & n.a. & $34.75 \pm 2.51$ & - \\
\hline 18 & 4d & 6 & $4-\mathrm{F}$ & 50 & n.a. & $55.25 \pm 3.66$ & - \\
\hline 19 & $4 e$ & 6 & 4-OMe & 33 & n.a. & $35.98 \pm 3.30$ & - \\
\hline 20 & $4 f$ & 6 & $4-\mathrm{CN}$ & 53 & n.a. & $29.56 \pm 4.37$ & - \\
\hline 21 & $4 g$ & 6 & $4-\mathrm{NO}_{2}$ & 55 & n.a. & $12.44 \pm 2.84$ & - \\
\hline 22 & 5 & 9 & $4-\mathrm{Cl}$ & 43 & n.a. & n.a. & - \\
\hline 23 & 6 & 10 & $4-\mathrm{Cl}$ & 33 & n.a. & n.a. & - \\
\hline 24 & $7 \mathbf{a}$ & \multicolumn{2}{|c|}{ Bis(10)-lophine } & 22 & n.a. & n.a. & - \\
\hline 25 & $7 \mathbf{b}$ & \multicolumn{2}{|c|}{ Bis(9)-lophine } & 42 & n.a. & n.a. & - \\
\hline 26 & 7c & \multicolumn{2}{|c|}{ Bis(8)-lophine } & 37 & $42.55 \pm 2.54$ & n.a. & - \\
\hline 27 & 7d & \multicolumn{2}{|c|}{ Bis(7)-lophine } & 29 & n.a. & n.a. & - \\
\hline 28 & 7e & \multicolumn{2}{|c|}{ Bis(6)-lophine } & 30 & n.a. & n.a. & - \\
\hline 29 & & \multicolumn{3}{|c|}{ Bis(7)-tacrine } & $4.12 \pm 0.70$ & $9.03 \pm 1.67$ & 2.2 \\
\hline
\end{tabular}

n.a. $=$ not active

a All yields refer to purified product.

b $\pm 95 \%$ Confidence limits.

$42.55 \mathrm{nM}$. We can infer that several new hybrids synthesized have targeted both the catalytic active site and the peripheral anionic site of AChE.

\section{Experimental protocols}

All melting points were determined in open glass capillaries using a Büchi M-565 apparatus. IR spectra were recorded on a Varian 640-IR spectrometer in $\mathrm{KBr}$ disks. ${ }^{1} \mathrm{H}$ NMR and ${ }^{13} \mathrm{C}$ NMR spectra were recorded in $\mathrm{CDCl}_{3}$ solution on a Varian VNMRS $300 \mathrm{MHz}$ spectrometer. The assignment of chemical shifts is based on standard NMR experiments $\left({ }^{1} \mathrm{H} ;{ }^{13} \mathrm{C}-\mathrm{APT} ;{ }^{1} \mathrm{H},{ }^{1} \mathrm{H}-\mathrm{COSY} ;{ }^{1} \mathrm{H},{ }^{13} \mathrm{C}-\right.$ HMQC). Chemical shifts $(\delta)$ are given in part per million from the peak of tetramethylsilane $(\delta=0.00 \mathrm{ppm})$ as internal standard in ${ }^{1} \mathrm{H}$ NMR or from the solvent peak of $\mathrm{CDCl}_{3}(\delta=77.23 \mathrm{ppm})$ in ${ }^{13} \mathrm{C}$ NMR; and multiplicities are given as $\mathrm{s}$ (singlet), $\mathrm{d}$ (doublet), $\mathrm{t}$ (triplet), qn (quintet), m (multiplet) or br (broad); coupling constants $(J)$ are given in Hz. High Resolution Mass Spectrometry with Eletrospray Ionization (HRMS-ESI) data on the positive mode was collected on a Micromass Q-Tof instrument from Waters (Manchester, UK). Samples were infused from a $100 \mu \mathrm{L}$ Hamilton syringe at flow rate range from 5 to $10 \mu \mathrm{L} / \mathrm{min}$, depending on the sample. The instrument settings were the following: capillary voltage $3000 \mathrm{~V}$, cone voltage $33 \mathrm{~V}$, extraction cone voltage $2.5 \mathrm{~V}$, desolvation gas temperature $100{ }^{\circ} \mathrm{C}$. Nitrogen was used as the desolvation gas. Methanol (Tedia HPLC grade) was used as solvent for the analysed samples and filtered prior to injection. Purification by column chromatography was carried out on silica gel 60 (70-230 mesh). Analytical thin layer chromatography (TLC) was conducted on aluminum plates with $0.2 \mathrm{~mm}$ of silica gel 60F-254 (Macherey-Nagel).

\subsection{Synthesis}

4.1.1. General procedure for the preparation of 9-alkylamino1,2,3,4-tetrahydroacridines

Intermediates 1a-e were prepared according to the previous literature procedure [32].

\subsubsection{General procedure for the preparation of hybrids $\mathbf{2 a}-\mathbf{g}, \mathbf{3 a}-\mathbf{g}$, $4 a-g, 5,6$}

A mixture of 9-alkylamino-1,2,3,4-tetrahydroacridines 1a-e (0.5 mmol), benzil $(0.5 \mathrm{mmol})$, aldehyde $(0.5 \mathrm{mmol})$, ammonium acetate $(0.5 \mathrm{mmol})$ and $\mathrm{InCl}_{3}(15 \mathrm{~mol} \%)$ in absolute ethanol $(1 \mathrm{~mL})$, placed in a round bottom flask fitted with a condenser and drying tube, was stirred at $78{ }^{\circ} \mathrm{C}$. After $48 \mathrm{~h}$, one more equivalent of benzil, aldehyde and ammonium acetate was added and the mixture was maintained at $78{ }^{\circ} \mathrm{C}$ for an additional $48 \mathrm{~h}$. The solvent was removed under reduced pressure, and the crude product purified by column chromatography (eluting with hexane-ethyl acetatetriethylamine, 60:39:1) to give the desired product.

4.1.2.1. 1,2,3,4-Tetrahydro-N-(8-(2,4,5-triphenyl-1H-imidazol-1-yl) octyl)acridin-9-amine (2a). Intermediate 1c was treated with benzil, benzaldehyde and ammonium acetate according to general procedure to give the desired product $2 \mathbf{a}$ as a yellow solid (55\% yield): m.p. $71-72{ }^{\circ} \mathrm{C}$; IR $(\mathrm{KBr}) \nu_{\max } / \mathrm{cm}^{-1}$ : 3346, 3058, 2927, 2854, 1579, 1500, 762, 698; ${ }^{1} \mathrm{H}$ NMR $\left(\mathrm{CDCl}_{3}\right) \delta 7.97-7.87(\mathrm{~m}, 2 \mathrm{H}), 7.73-$ $7.66(\mathrm{~m}, 2 \mathrm{H}), 7.58-7.28(\mathrm{~m}, 12 \mathrm{H}), 7.24-7.10$ (m, 3H), 4.00-3.80 (br, $1 \mathrm{H}), 3.87(\mathrm{t}, 2 \mathrm{H}, J=7.5 \mathrm{~Hz}), 3.40(\mathrm{t}, 2 \mathrm{H}, J=7.2 \mathrm{~Hz}), 3.12-3.00$ $(\mathrm{m}, 2 \mathrm{H}), 2.74-2.64(\mathrm{~m}, 2 \mathrm{H}), 1.98-1.84(\mathrm{~m}, 4 \mathrm{H}), 1.53$ (qn, 2H, $J=7.2 \mathrm{~Hz}), 1.41-1.12(\mathrm{~m}, 4 \mathrm{H}), 1.12-0.86(\mathrm{~m}, 6 \mathrm{H}) ;{ }^{13} \mathrm{C} \mathrm{NMR}\left(\mathrm{CDCl}_{3}\right)$ $\delta$ 158.6, 150.8, 147.8, 147.7, 137.8, 134.7, 131.7, 131.6, 131.1, 129.7, 129.3, $129.1,129.0,128.9,128.7,128.3,128.1,126.9,126.3,123.7,122.9$, 
120.4, 116.0, 49.6, 44.7, 34.2, 31.8, 30.3, 29.0, 28.6, 26.8, 26.1, 24.9, 23.2, 22.9; HRMS-ESI: calcd for $[\mathrm{M}-\mathrm{H}]^{+} 605.8463$, found 605.8474 .

4.1.2.2. $\mathrm{N}$-(8-(2-(4-Chlorophenyl)-4,5-diphenyl-1H-imidazol-1-yl) octyl)-1,2,3,4-tetrahydroacridin-9-amine (2b). Intermediate $\mathbf{1 c}$ was treated with benzil, 4-chlorobenzaldehyde and ammonium acetate according to general procedure to give the desired product $\mathbf{2 b}$ as a yellow solid (57\% yield): m.p. $62-63{ }^{\circ} \mathrm{C}$; IR $(K B r) \nu_{\max } / \mathrm{cm}^{-1}: 3367$, 3058, 2927, 2854, 1500, 835, 762, 698; ${ }^{1} \mathrm{H}$ NMR $\left(\mathrm{CDCl}_{3}\right) \delta$ 7.97-7.87 $(\mathrm{m}, 2 \mathrm{H}), 7.63(\mathrm{~d}, 2 \mathrm{H}, J=8.4 \mathrm{~Hz}), 7.58-7.28(\mathrm{~m}, 11 \mathrm{H}), 7.24-7.09(\mathrm{~m}$, $3 \mathrm{H}), 4.00-3.75(\mathrm{br}, 1 \mathrm{H}), 3.85(\mathrm{t}, 2 \mathrm{H}, J=7.5 \mathrm{~Hz}), 3.41(\mathrm{t}, 2 \mathrm{H}$, $J=7.3 \mathrm{~Hz}), 3.12-2.98(\mathrm{~m}, 2 \mathrm{H}), 2.76-2.60(\mathrm{~m}, 2 \mathrm{H}), 2.00-1.83(\mathrm{~m}$, $4 \mathrm{H}), 1.54$ (qn, $2 \mathrm{H}, J=7.3 \mathrm{~Hz}), 1.40-1.12(\mathrm{~m}, 4 \mathrm{H}), 1.12-0.86(\mathrm{~m}, 6 \mathrm{H})$; ${ }^{13} \mathrm{C}$ NMR $\left(\mathrm{CDCl}_{3}\right) \delta 158.6,150.8,147.7,146.6,138.1,135.0,134.5,131.5$, $131.1,130.6,130.2,130.1,129.2,129.0,128.9,128.8,128.4,128.2$, 126.9, 126.5, 123.7, 123.0, 120.4, 116.0, 49.6, 44.8, 34.2, 31.8, 30.4, 29.0, 28.6, 26.9, 26.2, 24.9, 23.2, 23.0; HRMS-ESI: calcd for [M - H] ${ }^{+}$ 640.2910, found 640.2910.

4.1.2.3. $\mathrm{N}-(8-(2-(3-C h l o r o p h e n y l)-4,5-d i p h e n y l-1 H$-imidazol-1-yl) octyl)- 1,2,3,4-tetrahydroacridin-9-amine (2c). Intermediate $\mathbf{1 c}$ was treated with benzil, 3-chlorobenzaldehyde and ammonium acetate according to general procedure to give the desired product $\mathbf{2 c}$ as a yellow solid (37\% yield): m.p. $43-44{ }^{\circ} \mathrm{C}$; IR $(K B r) \nu_{\max } / \mathrm{cm}^{-1}$ : 3367 , 3056, 2927, 2854, 1562, 1498, 767, 696; ${ }^{1} \mathrm{H} \mathrm{NMR}\left(\mathrm{CDCl}_{3}\right) \delta 7.97-7.86$ $(\mathrm{m}, 2 \mathrm{H}), 7.72(\mathrm{~s}, 1 \mathrm{H}), 7.60-7.27(\mathrm{~m}, 12 \mathrm{H}), 7.23-7.10(\mathrm{~m}, 3 \mathrm{H}), 4.00-3.80$ (br, $1 \mathrm{H}), 3.87(\mathrm{t}, 2 \mathrm{H}, J=7.5 \mathrm{~Hz}), 3.41(\mathrm{t}, 2 \mathrm{H}, J=7.1 \mathrm{~Hz}), 3.12-3.00(\mathrm{~m}$, $2 \mathrm{H}), 2.74-2.62(\mathrm{~m}, 2 \mathrm{H}), 2.00-1.83(\mathrm{~m}, 4 \mathrm{H}), 1.54(\mathrm{qn}, 2 \mathrm{H}, J=7.1 \mathrm{~Hz}$ ), $1.40-1.13(\mathrm{~m}, 4 \mathrm{H}), 1.13-0.88(\mathrm{~m}, 6 \mathrm{H}) ;{ }^{13} \mathrm{CNMR}\left(\mathrm{CDCl}_{3}\right) \delta 158.6,150.8$, 147.7, 146.2, 138.1, 134.7, 134.4, 133.4, 131.4, 131.1, 130.2, 130.0, 129.4, $129.2,129.0,128.9,128.8,128.4,128.2,127.2,126.9,126.5,123.7,122.9$, 120.4, 116.0, 49.6, 44.8, 34.2, 31.8, 30.4, 29.0, 28.6, 26.8, 26.1, 24.9, 23.2, 22.9; HRMS-ESI: calcd for $[\mathrm{M}-\mathrm{H}]^{+}$640.2910, found 640.2930.

4.1.2.4. $\mathrm{N}$-(8-(2-(4-Fluorophenyl)-4,5-diphenyl-1H-imidazol-1-yl) octyl)-1,2,3,4-tetrahydroacridin-9-amine (2d). Intermediate $\mathbf{1 c}$ was treated with benzil, 4-fluorobenzaldehyde and ammonium acetate according to general procedure to give the desired product $\mathbf{2 d}$ as a yellow solid (41\% yield): m.p. $57-58^{\circ} \mathrm{C}$; IR $(\mathrm{KBr}) \nu_{\max } / \mathrm{cm}^{-1}: 3380$, 3056, 2927, 1500, 1224, 843, 763, 698; ${ }^{1} \mathrm{H}$ NMR $\left(\mathrm{CDCl}_{3}\right) \delta 7.97-7.86$ (m, 2H), 7.72-7.63(m, 2H), 7.58-7.28 (m, 9H), 7.23-7.10 (m, 5H), 3.98-3.78 (br, $1 \mathrm{H}), 3.84(\mathrm{t}, 2 \mathrm{H}, J=7.6 \mathrm{~Hz}), 3.41(\mathrm{t}, 2 \mathrm{H}, J=7.2 \mathrm{~Hz})$, $3.12-2.98(\mathrm{~m}, 2 \mathrm{H}), 2.76-2.64(\mathrm{~m}, 2 \mathrm{H}), 2.00-1.84(\mathrm{~m}, 4 \mathrm{H}), 1.54(\mathrm{qn}$, $2 \mathrm{H}, J=7.2 \mathrm{~Hz}), 1.40-1.13(\mathrm{~m}, 4 \mathrm{H}), 1.13-0.88(\mathrm{~m}, 6 \mathrm{H}) ;{ }^{13} \mathrm{C} \mathrm{NMR}$ $\left(\mathrm{CDCl}_{3}\right) \delta 163.2(\mathrm{~d}, J=247.3 \mathrm{~Hz}), 158.6,150.8,147.7,146.8,137.8$, 134.6, 131.5, 131.3, 131.2, 131.1, 129.8, 129.2, 128.9, 128.8, 128.4, 128.2, $127.8(\mathrm{~d}, J=3.3 \mathrm{~Hz}), 126.9,126.4,123.7,122.9,120.4,116.0,115.8(\mathrm{~d}$, $J=21.4 \mathrm{~Hz}$ ), 49.6, 44.7, 34.2, 31.8, 30.3, 29.0, 28.6, 26.8, 26.2, 24.9, 23.2, 22.9; HRMS-ESI: calcd for $[\mathrm{M}-\mathrm{H}]^{+} 623.8367$, found 623.8380 .

4.1.2.5. 1,2,3,4-Tetrahydro-N-(8-(2-(4-methoxyphenyl)-4,5-diphenyl1H-imidazol-1-yl)octyl)acridin-9-amine (2e). Intermediate 1c was treated with benzil, 4-methoxybenzaldehyde and ammonium acetate according to general procedure to give the desired product $\mathbf{2 e}$ as a yellow solid (74\% yield): m.p. 56-57 ${ }^{\circ} \mathrm{C}$; IR $(\mathrm{KBr}) \nu_{\max } / \mathrm{cm}^{-1}$ : 3349, 3056, 2929, 1612, 1250, 1027, 837, 700; ${ }^{1} \mathrm{H}$ NMR $\left(\mathrm{CDCl}_{3}\right)$ $\delta$ 7.98-7.86 (m, 2H), $7.60(\mathrm{~d}, 2 \mathrm{H}, J=8.7 \mathrm{~Hz}), 7.58-7.28(\mathrm{~m}, 9 \mathrm{H})$, $7.24-7.08(\mathrm{~m}, 3 \mathrm{H}), 6.99(\mathrm{~d}, 2 \mathrm{H}, J=8.7 \mathrm{~Hz}), 3.96-3.78(\mathrm{~m}, 3 \mathrm{H}), 3.84$ $(\mathrm{s}, 3 \mathrm{H}), 3.41(\mathrm{t}, 2 \mathrm{H}, J=7.2 \mathrm{~Hz}), 3.12-3.00(\mathrm{~m}, 2 \mathrm{H}), 2.74-2.64(\mathrm{~m}, 2 \mathrm{H})$, $1.98-1.84(\mathrm{~m}, 4 \mathrm{H}), 1.54(\mathrm{qn}, 2 \mathrm{H}, J=7.2 \mathrm{~Hz}), 1.48-1.13(\mathrm{~m}, 4 \mathrm{H}), 1.13-$ $0.86(\mathrm{~m}, 6 \mathrm{H}) ;{ }^{13} \mathrm{C} \mathrm{NMR}\left(\mathrm{CDCl}_{3}\right) \delta 160.2,158.7,150.9,147.7,137.6$, $134.8,131.9,131.2,130.7,129.5,129.2,129.0,128.7,128.4,128.2$, 126.9, 126.3, 124.1, 123.7, 123.0, 120.4, 116.1, 114.2, 55.5, 49.6, 44.8, 34.3, 31.9, 30.4, 29.1, 28.7, 26.9, 26.2, 25.0, 23.2, 23.0; HRMS-ESI: calcd for $[\mathrm{M}-\mathrm{H}]^{+} 635.8725$, found 635.8731 .
4.1.2.6. 4-(1-(8-(1,2,3,4-Tetrahydroacridin-9-ylamino)octyl)-4,5diphenyl-1H-imidazol-2-yl)benzonitrile (2f). Intermediate 1c was treated with benzil, 4-cyanobenzaldehyde and ammonium acetate according to general procedure to give the desired product $2 \mathbf{2}$ as a yellow solid (33\% yield): m.p. 93-94 ${ }^{\circ} \mathrm{C} ; \mathrm{IR}(\mathrm{KBr}) \nu_{\max } / \mathrm{cm}^{-1}: 3402$, 3056, 2927, 2225, 1604, 1579, 771, 698; ${ }^{1} \mathrm{H}$ NMR $\left(\mathrm{CDCl}_{3}\right) \delta 8.02(\mathrm{~d}$, $1 \mathrm{H}, J=8.7 \mathrm{~Hz}), 7.96(\mathrm{~d}, 1 \mathrm{H}, J=8.4 \mathrm{~Hz}), 7.86(\mathrm{~d}, 2 \mathrm{H}, J=8.5 \mathrm{~Hz}), 7.77$ $(\mathrm{d}, 2 \mathrm{H}, J=8.5 \mathrm{~Hz}), 7.62-7.32(\mathrm{~m}, 9 \mathrm{H}), 7.24-7.12(\mathrm{~m}, 3 \mathrm{H}), 3.91(\mathrm{t}, 2 \mathrm{H}$, $J=7.6 \mathrm{~Hz}), 3.50(\mathrm{t}, 2 \mathrm{H}, J=7.3 \mathrm{~Hz}), 3.20-3.04(\mathrm{~m}, 2 \mathrm{H}), 2.75-2.60(\mathrm{~m}$, $2 \mathrm{H}), 2.00-1.83(\mathrm{~m}, 4 \mathrm{H}), 1.56(\mathrm{qn}, 2 \mathrm{H}, J=7.3 \mathrm{~Hz}), 1.44-1.15(\mathrm{~m}, 5 \mathrm{H})$, 1.15-0.84 (m, 6H); ${ }^{13} \mathrm{C}$ NMR $\left(\mathrm{CDCl}_{3}\right) \delta 157.3,151.8,146.0,145.6$, 138.9, 136.1, 134.2, 132.6, 131.1, 131.0, 129.6, 129.4, 129.3, 129.2, $128.4,126.9,126.8,124.1,123.2,119.5,118.8,115.0,112.3,49.4,45.1$, 33.1, 31.8, 30.5, 29.1, 28.7, 26.9, 26.2, 24.7, 23.0, 22.6; HRMS-ESI: calcd for $[\mathrm{M}-\mathrm{H}]^{+}$630.8561, found 630.8572 .

4.1.2.7. 1,2,3,4-Tetrahydro-N-(8-(2-(4-nitrophenyl)-4,5-diphenyl-1Himidazol-1-yl)octyl)acridin-9-amine (2g). Intermediate $\mathbf{1 c}$ was treated with benzil, 4-nitrobenzaldehyde and ammonium acetate according to general procedure to give the desired product $\mathbf{2} \mathbf{g}$ as a brown solid (39\% yield): m.p. $90-91{ }^{\circ} \mathrm{C}$; IR $(\mathrm{KBr}) \nu_{\max } / \mathrm{cm}^{-1}: 3422$, 3058, 2929, 2854, 1599, 1519, 1342, 698; ${ }^{1} \mathrm{H}$ NMR $\left(\mathrm{CDCl}_{3}\right) \delta 8.35(\mathrm{~d}$, $2 \mathrm{H}, J=7.8 \mathrm{~Hz}), 8.03(\mathrm{~d}, 1 \mathrm{H}, J=8.4 \mathrm{~Hz}), 7.99-7.88(\mathrm{~m}, 3 \mathrm{H}), 7.60-7.30$ $(\mathrm{m}, 9 \mathrm{H}), 7.25-7.12(\mathrm{~m}, 3 \mathrm{H}), 3.95(\mathrm{t}, 2 \mathrm{H}, J=7.6 \mathrm{~Hz}), 3.51(\mathrm{t}, 2 \mathrm{H}$, $J=7.2 \mathrm{~Hz}), 3.14-3.02(\mathrm{~m}, 2 \mathrm{H}), 2.70-2.58(\mathrm{~m}, 2 \mathrm{H}), 1.98-1.80(\mathrm{~m}$, $4 \mathrm{H}), 1.58$ (qn, $2 \mathrm{H}, J=7.2 \mathrm{~Hz}), 1.44-1.15(\mathrm{~m}, 5 \mathrm{H}), 1.15-0.80(\mathrm{~m}, 6 \mathrm{H})$; ${ }^{13} \mathrm{C}$ NMR $\left(\mathrm{CDCl}_{3}\right) \delta 157.1,151.9,147.7,145.8,145.2,139.1,137.9,134.2$ 131.4, 131.1, 131.0, 129.7, 129.4, 129.2, 128.4, 127.0, 126.9, 124.2, 124.1, $123.3,119.4,114.9,49.4,45.2,33.0,31.7,30.6,29.1,28.7,26.9,26.2$, 24.7, 23.0, 22.5; HRMS-ESI: calcd for $[\mathrm{M}-\mathrm{H}]^{+} 650.8439$, found 650.8471 .

4.1.2.8. 1,2,3,4-Tetrahydro-N-(7-(2,4,5-triphenyl-1H-imidazol-1-yl) heptyl)acridin-9-amine (3a). Intermediate $\mathbf{1 b}$ was treated with benzil, benzaldehyde and ammonium acetate according to general procedure to give the desired product 3a as a yellow solid $(54 \%$ yield): m.p. $61-62{ }^{\circ} \mathrm{C}$; IR $(\mathrm{KBr}) \nu_{\max } / \mathrm{cm}^{-1}$ : 3369, 3058, 2929, 2854, 1579, 1498, 762, 698; ${ }^{1} \mathrm{H}$ NMR $\left(\mathrm{CDCl}_{3}\right) \delta 7.94-7.86(\mathrm{~m}, 2 \mathrm{H}), 7.67(\mathrm{~d}$, $2 \mathrm{H}, J=6.9 \mathrm{~Hz}), 7.59-7.26(\mathrm{~m}, 12 \mathrm{H}), 7.22-7.09(\mathrm{~m}, 3 \mathrm{H}), 3.86(\mathrm{t}, 2 \mathrm{H}$, $J=7.5 \mathrm{~Hz}), 3.96-3.76(\mathrm{br}, 1 \mathrm{H}), 3.42-3.28(\mathrm{~m}, 2 \mathrm{H}), 3.14-3.00(\mathrm{~m}$, $2 \mathrm{H}), 2.70-2.56(\mathrm{~m}, 2 \mathrm{H}), 1.98-1.80(\mathrm{~m}, 4 \mathrm{H}), 1.43(\mathrm{qn}, 2 \mathrm{H}, J=7.3 \mathrm{~Hz})$, $1.38-1.24(\mathrm{~m}, 2 \mathrm{H}), 1.17-1.02(\mathrm{~m}, 2 \mathrm{H}), 1.01-0.84(\mathrm{~m}, 4 \mathrm{H}) ;{ }^{13} \mathrm{C} \mathrm{NMR}$ $\left(\mathrm{CDCl}_{3}\right) \delta 158.5,150.6,147.7,147.6,137.7,134.6,131.5,131.0,129.6$, 129.2, 129.1, 128.9, 128.8, 128.6, 128.2, 128.1, 126.8, 126.3, 123.6, $122.8,120.3,116.0,49.4,44.6,34.2,31.5,30.1,28.3,26.5,26.0,24.8$, 23.1, 22.8; HRMS-ESI: calcd for $[\mathrm{M}-\mathrm{H}]^{+} 591.8194$, found 591.8218 .

4.1.2.9. $\mathrm{N}$-(7-(2-(4-Chlorophenyl)-4,5-diphenyl-1H-imidazol-1-yl) heptyl)- 1,2,3,4-tetrahydroacridin-9-amine (3b). Intermediate $\mathbf{1 b}$ was treated with benzil, 4-chlorobenzaldehyde and ammonium acetate according to general procedure to give the desired product 3b as a yellow solid (33\% yield): m.p. $64-65{ }^{\circ} \mathrm{C}$; IR $(\mathrm{KBr}) \nu_{\max } / \mathrm{cm}^{-1}$ : 3367, 3057, 2927, 2854, 1500, 835, 762, 698; ${ }^{1} \mathrm{H}$ NMR $\left(\mathrm{CDCl}_{3}\right)$ $\delta$ 7.94-7.87 (m, 2H), $7.63(\mathrm{~d}, 2 \mathrm{H}, J=8.7 \mathrm{~Hz}), 7.58-7.28(\mathrm{~m}, 11 \mathrm{H})$, $7.23-7.10(\mathrm{~m}, 3 \mathrm{H}), 3.86(\mathrm{t}, 2 \mathrm{H}, J=7.5 \mathrm{~Hz}), 3.34(\mathrm{t}, 2 \mathrm{H}, J=7.2 \mathrm{~Hz})$, $3.12-3.00(\mathrm{~m}, 2 \mathrm{H}), 2.73-2.60(\mathrm{~m}, 2 \mathrm{H}), 1.98-1.84(\mathrm{~m}, 4 \mathrm{H}), 1.48$ (qn, $2 \mathrm{H}, J=7.2 \mathrm{~Hz}), 1.40-1.24(\mathrm{~m}, 3 \mathrm{H}), 1.20-1.05(\mathrm{~m}, 2 \mathrm{H}), 1.04-0.88(\mathrm{~m}$, $4 \mathrm{H}) ;{ }^{13} \mathrm{C} \mathrm{NMR}\left(\mathrm{CDCl}_{3}\right) \delta 158.7,150.8,147.7,146.6,138.1,135.0,134.5$, $131.5,131.1,130.6,130.2,130.1,129.2,129.1,129.0,128.9,128.8,128.4$, 128.3, 126.9, 126.5, 123.8, 122.9, 120.4, 116.1, 49.5, 44.8, 34.2, 31.7, 30.4, 28.5, 26.7, 26.2, 25.0, 23.2, 23.0; HRMS-ESI: calcd for $[\mathrm{M}-\mathrm{H}]^{+}$ 626.2641 , found 626.2669 .

4.1.2.10. $\mathrm{N}-(7-(2-(3-C h l o r o p h e n y l)-4,5-d i p h e n y l-1 H$-imidazol-1-yl) heptyl)-1,2,3,4-tetrahydroacridin-9-amine (3c). Intermediate $\mathbf{1 b}$ 
was treated with benzil, 3-chlorobenzaldehyde and ammonium acetate according to general procedure to give the desired product 3c as a yellow solid (42\% yield): m.p. 59-60 ${ }^{\circ} \mathrm{C}$; IR $(\mathrm{KBr}) \nu_{\max } / \mathrm{cm}^{-1}$ : 3367, 3056, 2927, 2854, 1562, 1498, 767, 696; ${ }^{1} \mathrm{H}$ NMR $\left(\mathrm{CDCl}_{3}\right)$ $\delta$ 7.95-7.86 (m, 2H), $7.72(\mathrm{~s}, 1 \mathrm{H}), 7.60-7.27(\mathrm{~m}, 12 \mathrm{H}), 7.23-7.10(\mathrm{~m}$, $3 \mathrm{H}), 4.00-3.78(\mathrm{br}, 1 \mathrm{H}), 3.87(\mathrm{t}, 2 \mathrm{H}, J=7.5 \mathrm{~Hz}), 3.35(\mathrm{t}, 2 \mathrm{H}$, $J=7.1 \mathrm{~Hz}), 3.14-3.00(\mathrm{~m}, 2 \mathrm{H}), 2.76-2.58(\mathrm{~m}, 2 \mathrm{H}), 2.00-1.82(\mathrm{~m}$, $4 \mathrm{H}), 1.47(\mathrm{qn}, 2 \mathrm{H}, J=7.1 \mathrm{~Hz}), 1.39-1.24(\mathrm{~m}, 2 \mathrm{H}), 1.20-1.15(\mathrm{~m}, 2 \mathrm{H})$, 1.15-0.88 (m, 4H); ${ }^{13} \mathrm{C}$ NMR $\left(\mathrm{CDCl}_{3}\right) \delta 158.6,150.7,147.7,146.2$, 138.1, 134.7, 134.4, 133.3, 131.3, 131.1, 130.1, 130.0, 129.4, 129.2, 129.0, $128.9,128.8,128.4,128.2,127.2,126.9,126.5,123.7,122.9,120.4$, 116.1, 49.5, 44.7, 34.2, 31.7, 30.3, 28.4, 26.6, 26.1, 24.9, 23.2, 22.9; HRMS-ESI: calcd for $[\mathrm{M}-\mathrm{H}]^{+} 626.2641$, found 626.2654 .

4.1.2.11. N-(7-(2-(4-Fluorophenyl)-4,5-diphenyl-1H-imidazol-1-yl) heptyl)-1,2,3,4-tetrahydroacridin-9-amine (3d). Intermediate $\mathbf{1 b}$ was treated with benzil, 4-fluorobenzaldehyde and ammonium acetate according to general procedure to give the desired product 3d as a yellow solid (52\% yield): m.p. $61-62{ }^{\circ} \mathrm{C}$; IR $(\mathrm{KBr}) \nu_{\max } / \mathrm{cm}^{-1}$ : $3361,3056,2929,1500,1224,843,761,698 ;{ }^{1} \mathrm{H}$ NMR $\left(\mathrm{CDCl}_{3}\right) \delta 7.95-$ $7.86(\mathrm{~m}, 2 \mathrm{H}), 7.70-7.62(\mathrm{~m}, 2 \mathrm{H}), 7.59-7.28(\mathrm{~m}, 9 \mathrm{H}), 7.24-7.09(\mathrm{~m}$, $5 \mathrm{H}), 3.96-3.76(\mathrm{br}, 1 \mathrm{H}), 3.84(\mathrm{t}, 2 \mathrm{H}, J=7.5 \mathrm{~Hz}), 3.42-3.28(\mathrm{~m}, 2 \mathrm{H})$, $3.14-2.98(\mathrm{~m}, 2 \mathrm{H}), 2.74-2.58(\mathrm{~m}, 2 \mathrm{H}), 2.00-1.80(\mathrm{~m}, 4 \mathrm{H}), 1.47$ (qn, $2 \mathrm{H}, J=7.2 \mathrm{~Hz}), 1.38-1.24(\mathrm{~m}, 2 \mathrm{H}), 1.19-1.05(\mathrm{~m}, 2 \mathrm{H}), 1.05-0.84(\mathrm{~m}$, $4 \mathrm{H}) ;{ }^{13} \mathrm{C} \mathrm{NMR}\left(\mathrm{CDCl}_{3}\right) \delta 163.1(\mathrm{~d}, J=247.2 \mathrm{~Hz}), 158.6,150.7,147.6$, 146.7, 137.8, 134.5, 131.4, 131.2, 131.1, 131.0, 129.7, 129.2, 128.9, 128.8, $128.3,128.2,127.8$ (d, $J=3.3 \mathrm{~Hz}), 126.8,126.4,123.7,122.9,120.3$, $116.1,115.8$ (d, $J=22.0 \mathrm{~Hz}$ ), 49.4, 44.6, 34.2, 31.6, 30.2, 28.4, 26.5, 26.1, 24.9, 23.1, 22.9; HRMS-ESI: calcd for $[\mathrm{M}-\mathrm{H}]^{+} 609.8098$, found 609.8119 .

4.1.2.12. 1,2,3,4-Tetrahydro-N-(7-(2-(4-methoxyphenyl)-4,5-diphenyl1 H-imidazol-1-yl)heptyl)acridin-9-amine (3e). Intermediate $\mathbf{1 b}$ was treated with benzil, 4-methoxybenzaldehyde and ammonium acetate according to general procedure to give the desired product $3 \mathbf{e}$ as a yellow solid (44\% yield): m.p. $67-68^{\circ} \mathrm{C}$; IR $(\mathrm{KBr}) \nu_{\max } / \mathrm{cm}^{-1}: 3378$, 3056, 2929, 1500, 1249, 1027, 771, 700; ${ }^{1} \mathrm{H}$ NMR $\left(\mathrm{CDCl}_{3}\right) \delta$ 7.94-7.86 (m, 2H), $7.60(\mathrm{~d}, 2 \mathrm{H}, J=9.0 \mathrm{~Hz}), 7.56-7.28(\mathrm{~m}, 9 \mathrm{H}), 7.22-7.08$ $(\mathrm{m}, 3 \mathrm{H}), 6.98(\mathrm{~d}, 2 \mathrm{H}, J=9.0 \mathrm{~Hz}), 3.90-3.78(\mathrm{~m}, 3 \mathrm{H}), 3.82(\mathrm{~s}, 3 \mathrm{H})$, $3.35(\mathrm{t}, 2 \mathrm{H}, J=7.2 \mathrm{~Hz}), 3.10-3.00(\mathrm{~m}, 2 \mathrm{H}), 2.70-2.62(\mathrm{~m}, 2 \mathrm{H}), 1.96-$ $1.84(\mathrm{~m}, 4 \mathrm{H}), 1.47$ (qn, 2H, $J=7.2 \mathrm{~Hz}), 1.31$ (qn, 2H, $=7.2 \mathrm{~Hz}), 1.17-$ $1.04(\mathrm{~m}, 2 \mathrm{H}), 1.04-0.88(\mathrm{~m}, 4 \mathrm{H}) ;{ }^{13} \mathrm{C} \mathrm{NMR}\left(\mathrm{CDCl}_{3}\right) \delta 160.2,158.7$, 150.8, 147.7, 147.6, 137.7, 134.8, 131.8, 131.2, 130.7, 129.5, 129.1, 129.0, 128.7, 128.4, 128.2, 126.9, 126.3, 124.1, 123.7, 122.9, 120.4, 116.1, 114.2, 55.5, 49.5, 44.7, 34.2, 31.7, 30.3, 28.5, 26.6, 26.2, 25.0, 23.2, 23.0; HRMS-ESI: calcd for $[\mathrm{M}-\mathrm{H}]^{+} 621.8457$, found 621.8468 .

4.1.2.13. 4-(1-(7-(1,2,3,4-Tetrahydroacridin-9-ylamino $)$ heptyl)-4,5diphenyl-1H-imidazol-2-yl)benzonitrile (3f). Intermediate $\mathbf{1 b}$ was treated with benzil, 4-cyanobenzaldehyde and ammonium acetate according to general procedure to give the desired product $\mathbf{3 f}$ as a yellow solid (53\% yield): m.p. $78-79{ }^{\circ} \mathrm{C}$; IR $(\mathrm{KBr}) \nu_{\max } / \mathrm{cm}^{-1}: 3400$, 3056, 2929, 2225, 1606, 1500, 771, 700; ${ }^{1} \mathrm{H} \mathrm{NMR}\left(\mathrm{CDCl}_{3}\right) \delta 7.95(\mathrm{~d}, 1 \mathrm{H}$, $J=8.7 \mathrm{~Hz}), 7.91(\mathrm{~d}, 1 \mathrm{H}, J=8.4 \mathrm{~Hz}), 7.85(\mathrm{~d}, 2 \mathrm{H}, J=8.5 \mathrm{~Hz}), 7.77(\mathrm{~d}, 2 \mathrm{H}$, $J=8.5 \mathrm{~Hz}$ ), 7.59-7.30 (m, 9H), 7.24-7.12 (m, 3H), 4.20-3.80 (br, 1H), $3.91(\mathrm{t}, 2 \mathrm{H}, J=7.5 \mathrm{~Hz}), 3.41(\mathrm{t}, 2 \mathrm{H}, J=7.2 \mathrm{~Hz}), 3.14-3.02(\mathrm{~m}, 2 \mathrm{H}), 2.72-$ $2.60(\mathrm{~m}, 2 \mathrm{H}), 1.98-1.83(\mathrm{~m}, 4 \mathrm{H}), 1.50$ (qn, $2 \mathrm{H}, J=7.2 \mathrm{~Hz}), 1.33$ (qn, $2 \mathrm{H}$, $J=7.5 \mathrm{~Hz}), 1.20-1.06(\mathrm{~m}, 2 \mathrm{H}), 1.06-0.88(\mathrm{~m}, 4 \mathrm{H}) ;{ }^{13} \mathrm{C} \mathrm{NMR}\left(\mathrm{CDCl}_{3}\right)$ $\delta 158.1,151.2,146.9,145.6,138.9,136.0,134.2,132.6,131.1,131.0,129.5$, 129.3, 129.1, 128.8, 128.3, 126.9, 126.8, 123.9, 123.0, 120.0, 118.7, 115.7, $112.3,49.4,45.1,33.7,31.7,30.5,28.6,26.7,26.2,24.8,23.1,22.8$; HRMS-ESI: calcd for $[\mathrm{M}-\mathrm{H}]^{+} 616.8292$, found 616.8281 .

4.1.2.14. 1,2,3,4-Tetrahydro-N-(7-(2-(4-nitrophenyl)-4,5-diphenyl1H-imidazol-1-yl)heptyl)acridin-9-amine (3g). Intermediate $\mathbf{1 b}$ was treated with benzil, 4-nitrobenzaldehyde and ammonium acetate according to general procedure to give the desired product $\mathbf{3 g}$ as a brown solid (44\% yield): m.p. $84-85^{\circ} \mathrm{C}$; IR $(\mathrm{KBr}) \nu_{\max } / \mathrm{cm}^{-1}$ : 3411 , 3058, 2929, 2856, 1599, 1519, 1342, 696; ${ }^{1} \mathrm{H}$ NMR $\left(\mathrm{CDCl}_{3}\right) \delta 8.35$ (d, $2 \mathrm{H}, J=8.4 \mathrm{~Hz}), 7.86-7.97(\mathrm{~m}, 4 \mathrm{H}), 7.28-7.58(\mathrm{~m}, 9 \mathrm{H}), 7.24-7.12(\mathrm{~m}$, $3 \mathrm{H}), 3.94(\mathrm{t}, 2 \mathrm{H}, J=7.5 \mathrm{~Hz}), 3.37(\mathrm{t}, 2 \mathrm{H}, J=7.2 \mathrm{~Hz}), 3.12-2.59(\mathrm{~m}$, $2 \mathrm{H}), 2.72-2.60(\mathrm{~m}, 2 \mathrm{H}), 1.98-1.83(\mathrm{~m}, 4 \mathrm{H}), 1.49(\mathrm{qn}, 2 \mathrm{H}, J=7.2 \mathrm{~Hz})$, $1.42-1.22(\mathrm{~m}, 3 \mathrm{H}), 1.20-1.08(\mathrm{~m}, 2 \mathrm{H}), 1.08-0.90(\mathrm{~m}, 4 \mathrm{H}) ;{ }^{13} \mathrm{C} \mathrm{NMR}$ $\left(\mathrm{CDCl}_{3}\right) \delta 158.6,150.9,147.8,147.5,145.2,139.2,137.9,134.1,131.4$, 131.1, 131.0, 129.7, 129.4, 129.2, 128.8, 128.6, 128.4, 127.0, 126.9, $124.2,123.8,122.9,120.3,116.1,49.5,45.2,34.1,31.7,30.6,28.6,26.7$, 26.3, 25.0, 23.2, 22.9; HRMS-ESI: calcd for $[\mathrm{M}-\mathrm{H}]^{+}$636.8170, found 636.8161 .

4.1.2.15. 1,2,3,4-Tetrahydro-N-(6-(2,4,5-triphenyl-1H-imidazol-1-yl) hexyl)acridin-9-amine (4a). Intermediate $\mathbf{1 a}$ was treated with benzil, benzaldehyde and ammonium acetate according to general procedure to give the desired product $4 \mathbf{a}$ as a yellow solid $(53 \%$ yield): m.p. $62-63{ }^{\circ} \mathrm{C}$; IR $(\mathrm{KBr}) \nu_{\max } / \mathrm{cm}^{-1}$ : 3369, 3058, 2929, 2856, 1579, 1500, 764, 698; ${ }^{1} \mathrm{H}$ NMR $\left(\mathrm{CDCl}_{3}\right) \delta 7.91(\mathrm{~d}, 1 \mathrm{H}, J=8.4 \mathrm{~Hz}), 7.84$ $(\mathrm{d}, 1 \mathrm{H}, J=8.4 \mathrm{~Hz}), 7.66(\mathrm{~d}, 2 \mathrm{H}, J=7.3 \mathrm{~Hz}), 7.59-7.25(\mathrm{~m}, 12 \mathrm{H}), 7.23-$ $7.08(\mathrm{~m}, 3 \mathrm{H}), 3.86(\mathrm{t}, 2 \mathrm{H}, J=7.3 \mathrm{~Hz}), 3.79(\mathrm{br}, 1 \mathrm{H}), 3.32-3.20(\mathrm{~m}, 2 \mathrm{H})$, $3.12-3.00(\mathrm{~m}, 2 \mathrm{H}), 2.68-2.52(\mathrm{~m}, 2 \mathrm{H}), 1.98-1.78(\mathrm{~m}, 4 \mathrm{H}), 1.44-1.20$ $(\mathrm{m}, 4 \mathrm{H}), 1.10-0.88(\mathrm{~m}, 4 \mathrm{H}) ;{ }^{13} \mathrm{C} \mathrm{NMR}\left(\mathrm{CDCl}_{3}\right) \delta 158.3,150.6,147.6$, 147.4, 137.7, 134.5, 131.4, 130.9, 129.6, 129.1, 129.0, 128.8, 128.7, 128.6, 128.2, 128.0, 126.7, 126.2, 123.5, 122.8, 120.2, 115.9, 49.1, 44.4, 34.0, $31.2,30.0,25.8,25.7,24.7,23.0,22.7$; HRMS-ESI: calcd for $[\mathrm{M}-\mathrm{H}]^{+}$ 577.7925, found 577.7941 .

4.1.2.16. $N$-(6-(2-(4-Chlorophenyl)-4,5-diphenyl-1H-imidazol-1-yl) hexyl)-1,2,3,4-tetrahydroacridin-9-amine (4b). Intermediate 1a was treated with benzil, 4-chlorobenzaldehyde and ammonium acetate according to general procedure to give the desired product $\mathbf{4 b}$ as a yellow solid (37\% yield): m.p. $71-72{ }^{\circ} \mathrm{C}$; IR $(\mathrm{KBr}) \nu_{\max } / \mathrm{cm}^{-1}$ : 3377 , 3056, 2931, 2856, 1500, 835, 761, 698; ${ }^{1} \mathrm{H} \mathrm{NMR}\left(\mathrm{CDCl}_{3}\right) \delta 7.90(\mathrm{~d}, 1 \mathrm{H}$, $J=8.4 \mathrm{~Hz}), 7.85(\mathrm{~d}, 1 \mathrm{H}, J=8.4 \mathrm{~Hz}), 7.62(\mathrm{~d}, 2 \mathrm{H}, J=8.4 \mathrm{~Hz}), 7.56-7.27$ (m, 11H), 7.23-7.10 (m, 3H), $3.85(\mathrm{t}, 2 \mathrm{H}, J=7.5 \mathrm{~Hz}), 3.79(\mathrm{br}, 1 \mathrm{H})$, $3.28(\mathrm{t}, 2 \mathrm{H}, \mathrm{J}=7.2 \mathrm{~Hz}), 3.10-3.00(\mathrm{~m}, 2 \mathrm{H}), 2.68-2.58(\mathrm{~m}, 2 \mathrm{H}), 1.98-$ $1.80(\mathrm{~m}, 4 \mathrm{H}), 1.44-1.25(\mathrm{~m}, 4 \mathrm{H}), 1.10-0.88(\mathrm{~m}, 4 \mathrm{H}) ;{ }^{13} \mathrm{C} \mathrm{NMR}\left(\mathrm{CDCl}_{3}\right)$ $\delta$ 158.6, 150.7, 147.6, 146.5, 138.1, 135.0, 134.4, 131.4, 131.0, 130.5, 130.1, 130.0, 129.2, 129.0, 128.9, 128.8, 128.4, 128.2, 126.9, 126.5, 123.7, 122.8, 120.4, 116.2, 49.3, 44.7, 34.2, 31.4, 30.3, 26.1, 26.0, 24.9, 23.1, 22.9; HRMS-ESI: calcd for $[\mathrm{M}-\mathrm{H}]^{+} 612.2373$, found 612.2393 .

4.1.2.17. $N$-(6-(2-(3-Chlorophenyl)-4,5-diphenyl-1H-imidazol-1-yl) hexyl)-1,2,3,4-tetrahydroacridin-9-amine (4c). Intermediate 1a was treated with benzil, 3-chlorobenzaldehyde and ammonium acetate according to general procedure to give the desired product $\mathbf{4 c}$ as a yellow solid (29\% yield): m.p. $62-63^{\circ} \mathrm{C}$; IR $(K B r) \nu_{\max } / \mathrm{cm}^{-1}: 3361$, 3056, 2929, 2856, 1562, 1498, 767, 696; ${ }^{1} \mathrm{H} \mathrm{NMR}\left(\mathrm{CDCl}_{3}\right) \delta 7.90(\mathrm{~d}$, $1 \mathrm{H}, J=8.4 \mathrm{~Hz}), 7.86(\mathrm{~d}, 1 \mathrm{H}, J=8.4 \mathrm{~Hz}), 7.71(\mathrm{~s}, 1 \mathrm{H}), 7.60-7.27(\mathrm{~m}$, $12 \mathrm{H}), 7.23-7.10(\mathrm{~m}, 3 \mathrm{H}), 3.88(\mathrm{t}, 2 \mathrm{H}, J=7.5 \mathrm{~Hz}), 3.79(\mathrm{br}, 1 \mathrm{H}), 3.28(\mathrm{t}$, $2 \mathrm{H}, J=7.2 \mathrm{~Hz}), 3.12-2.98(\mathrm{~m}, 2 \mathrm{H}), 2.72-2.58(\mathrm{~m}, 2 \mathrm{H}), 1.98-1.80(\mathrm{~m}$, $4 \mathrm{H}), 1.48-1.25(\mathrm{~m}, 4 \mathrm{H}), 1.12-0.91(\mathrm{~m}, 4 \mathrm{H}) ;{ }^{13} \mathrm{C} \mathrm{NMR}\left(\mathrm{CDCl}_{3}\right) \delta 158.6$, 150.7, 147.6, 146.2, 138.2, 134.7, 134.4, 133.3, 131.3, 131.0, 130.1, 130.0, $129.3,129.2,129.0,128.9,128.4,128.2,127.2,126.9,126.5,123.7$, 122.8, 120.4, 116.2, 49.3, 44.6, 34.2, 31.5, 30.3, 26.1, 26.0, 24.9, 23.1, 22.9; HRMS-ESI: calcd for $[\mathrm{M}-\mathrm{H}]^{+} 612.2373$, found 612.2372 .

4.1.2.18. $\mathrm{N}$-(6-(2-(4-Fluorophenyl)-4,5-diphenyl-1H-imidazol-1-yl) hexyl)-1,2,3,4-tetrahydroacridin-9-amine (4d). Intermediate 1a was treated with benzil, 4-fluorobenzaldehyde and ammonium acetate according to general procedure to give the desired product $4 \mathbf{d}$ as a yellow solid (50\% yield): m.p. $64-65{ }^{\circ} \mathrm{C}$; IR $(\mathrm{KBr}) \nu_{\max } / \mathrm{cm}^{-1}: 3355$, $3056,2929,1500,1224,843,764,698 ;{ }^{1} \mathrm{H} \mathrm{NMR}\left(\mathrm{CDCl}_{3}\right) \delta 7.89(\mathrm{~d}, 1 \mathrm{H}$, 
$J=8.7 \mathrm{~Hz}), 7.85(\mathrm{~d}, 1 \mathrm{H}, J=8.4 \mathrm{~Hz}), 7.70-7.60(\mathrm{~m}, 2 \mathrm{H}), 7.58-7.27(\mathrm{~m}$, 9H), 7.23-7.08 (m, 5H), $3.84(\mathrm{t}, 2 \mathrm{H}, J=7.5 \mathrm{~Hz}), 3.77(\mathrm{br}, 1 \mathrm{H}), 3.38-$ $3.20(\mathrm{~m}, 2 \mathrm{H}), 3.12-2.98(\mathrm{~m}, 2 \mathrm{H}), 2.68-2.54(\mathrm{~m}, 2 \mathrm{H}), 1.98-1.80(\mathrm{~m}$, $4 \mathrm{H}), 1.44-1.20(\mathrm{~m}, 4 \mathrm{H}), 1.10-0.88(\mathrm{~m}, 4 \mathrm{H}) ;{ }^{13} \mathrm{C} \mathrm{NMR}\left(\mathrm{CDCl}_{3}\right) \delta 163.1$ $(\mathrm{d}, J=247.7 \mathrm{~Hz}), 158.7,150.6,147.7,146.7,137.9,134.5,131.5,131.2$, 131.1, 131.0, 129.7, 129.2, 129.0, 128.8, 128.3, 128.2, 127.8 (d, $J=3.3 \mathrm{~Hz}), 126.8,126.4,123.7,122.8,120.4,116.2,115.8(\mathrm{~d}$, $J=22.0 \mathrm{~Hz}$ ), 49.3, 44.6, 34.2, 31.4, 30.3, 26.1, 26.0, 24.9, 23.1, 22.9; HRMS-ESI: calcd for [M $-\mathrm{H}]^{+}$595.7830, found 595.7809.

4.1.2.19. 1,2,3,4-Tetrahydro-N-(6-(2-(4-methoxyphenyl)-4,5-diphenyl1 H-imidazol-1-yl)hexyl)acridin-9-amine (4e). Intermediate $\mathbf{1 a}$ was treated with benzil, 4-methoxybenzaldehyde and ammonium acetate according to general procedure to give the desired product $4 \mathbf{e}$ as a yellow solid (33\% yield): m.p. $68-69^{\circ} \mathrm{C}$; IR $(\mathrm{KBr}) \nu_{\max } / \mathrm{cm}^{-1}: 3365$, 3056, 2931, 1500, 1249, 1028, 764, 698; ${ }^{1} \mathrm{H} \mathrm{NMR}\left(\mathrm{CDCl}_{3}\right) \delta 7.89(\mathrm{~d}, 1 \mathrm{H}$, $J=8.7 \mathrm{~Hz}), 7.85(\mathrm{~d}, 1 \mathrm{H}, J=8.4 \mathrm{~Hz}), 7.60(\mathrm{~d}, 2 \mathrm{H}, J=8.7 \mathrm{~Hz}), 7.34-7.55$ $(\mathrm{m}, 8 \mathrm{H}), 7.34-7.26(\mathrm{~m}, 1 \mathrm{H}), 7.21-7.07(\mathrm{~m}, 3 \mathrm{H}), 6.96(\mathrm{~d}, 2 \mathrm{H}$, $J=8.7 \mathrm{~Hz}$ ), 3.90-3.70 (br, $1 \mathrm{H}), 3.84(\mathrm{t}, 2 \mathrm{H}, J=7.5 \mathrm{~Hz}), 3.79(\mathrm{~s}, 3 \mathrm{H})$, $3.26(\mathrm{t}, 2 \mathrm{H}, J=7.2 \mathrm{~Hz}), 3.10-3.00(\mathrm{~m}, 2 \mathrm{H}), 2.68-2.58(\mathrm{~m}, 2 \mathrm{H}), 1.96-$ $1.82(\mathrm{~m}, 4 \mathrm{H}), 1.44-1.23(\mathrm{~m}, 4 \mathrm{H}), 1.10-0.88(\mathrm{~m}, 4 \mathrm{H}) ;{ }^{13} \mathrm{C} \mathrm{NMR}\left(\mathrm{CDCl}_{3}\right)$ $\delta$ 160.1, 158.6, 150.6, 147.7, 147.6, 137.6, 134.7, 131.7, 131.0, 130.5, 129.4, 129.1, 128.9, 128.6, 128.3, 128.1, 126.8, 126.2, 124.0, 123.6, 122.8, 120.3, 116.1, 114.1, 55.4, 49.3, 44.5, 34.2, 31.4, 30.1, 26.0, 25.9, 24.8, 23.1, 22.9; HRMS-ESI: calcd for $[\mathrm{M}-\mathrm{H}]^{+} 607.8188$, found 607.8179 .

4.1.2.20. 4-(1-(6-(1,2,3,4-Tetrahydroacridin-9-ylamino)hexyl)-4,5diphenyl-1H-imidazol-2-yl)benzonitrile (4f). Intermediate 1a was treated with benzil, 4-cyanobenzaldehyde and ammonium acetate according to general procedure to give the desired product $\mathbf{4 f}$ as a yellow solid (53\% yield): m.p. $74-75^{\circ} \mathrm{C}$; IR $(\mathrm{KBr}) \nu_{\max } / \mathrm{cm}^{-1}: 3384$, 3056, 2927, 2225, 1604, 1498, 769, 696; ${ }^{1} \mathrm{H}$ NMR $\left(\mathrm{CDCl}_{3}\right) \delta 7.90(\mathrm{~d}$, $1 \mathrm{H}, J=8.7 \mathrm{~Hz}), 7.85(\mathrm{~d}, 1 \mathrm{H}, J=7.8 \mathrm{~Hz}), 7.84(\mathrm{~d}, 2 \mathrm{H}, J=8.5 \mathrm{~Hz}), 7.75$ $(\mathrm{d}, 2 \mathrm{H}, J=8.5 \mathrm{~Hz}), 7.58-7.28(\mathrm{~m}, 9 \mathrm{H}), 7.24-7.12(\mathrm{~m}, 3 \mathrm{H}), 3.91(\mathrm{t}, 2 \mathrm{H}$, $J=7.6 \mathrm{~Hz}), 3.79(\mathrm{br}, 1 \mathrm{H}), 3.30(\mathrm{t}, 2 \mathrm{H}, J=7.3 \mathrm{~Hz}), 3.12-3.00(\mathrm{~m}, 2 \mathrm{H})$, $2.68-2.58(\mathrm{~m}, 2 \mathrm{H}), 1.98-1.82(\mathrm{~m}, 4 \mathrm{H}), 1.48-1.24(\mathrm{~m}, 4 \mathrm{H}), 1.12-0.90$ $(\mathrm{m}, 4 \mathrm{H}) ;{ }^{13} \mathrm{C}$ NMR $\left(\mathrm{CDCl}_{3}\right) \delta 158.7,150.7,147.6,145.6,138.9,136.0$, 134.1, 132.6, 131.1, 131.0, 129.5, 129.4, 129.2, 129.0, 128.5, 128.3, $126.9,126.8,123.8,122.8,120.4,118.7,116.3,112.4,49.3,45.0,34.2$, $31.5,30.5,26.2,26.1,25.0,23.2,22.9$; HRMS-ESI: calcd for $[\mathrm{M}-\mathrm{H}]^{+}$ 602.8023 , found 602.8043 .

4.1.2.21. 1,2,3,4-Tetrahydro-N-(6-(2-(4-nitrophenyl)-4,5-diphenyl1H-imidazol-1-yl)hexyl)acridin-9-amine (4g). Intermediate $1 \mathbf{a}$ was treated with benzil, 4-nitrobenzaldehyde and ammonium acetate according to general procedure to give the desired product $4 \mathrm{~g}$ as a brown solid (55\% yield): m.p. $73-74{ }^{\circ} \mathrm{C}$; IR $(\mathrm{KBr}) \nu_{\max } /$ $\mathrm{cm}^{-1}$ : 3417, 3058, 2929, 2856, 1599, 1519, 1340, 696; ${ }^{1} \mathrm{H}$ NMR $\left(\mathrm{CDCl}_{3}\right) \delta 8.32(\mathrm{~d}, 2 \mathrm{H}, J=8.7 \mathrm{~Hz}), 7.98-7.87(\mathrm{~m}, 3 \mathrm{H}), 7.85(\mathrm{~d}, 1 \mathrm{H}$, $J=8.1 \mathrm{~Hz}), 7.58-7.27(\mathrm{~m}, 9 \mathrm{H}), 7.25-7.12(\mathrm{~m}, 3 \mathrm{H}), 3.94(\mathrm{t}, 2 \mathrm{H}$, $J=7.5 \mathrm{~Hz}), 3.73(\mathrm{br}, 1 \mathrm{H}), 3.29(\mathrm{t}, 2 \mathrm{H}, J=7.0 \mathrm{~Hz}), 3.12-2.98(\mathrm{~m}$, $2 \mathrm{H}), 2.72-2.56(\mathrm{~m}, 2 \mathrm{H}), 1.98-1.80(\mathrm{~m}, 4 \mathrm{H}), 1.50-1.28(\mathrm{~m}, 4 \mathrm{H})$, $1.14-0.90(\mathrm{~m}, 4 \mathrm{H}) ;{ }^{13} \mathrm{C} \mathrm{NMR}\left(\mathrm{CDCl}_{3}\right) \delta 158.6,150.7,147.7,147.5$, 145.2, 139.1, 137.7, 134.1, 131.4, 131.0, 130.9, 129.6, 129.4, 129.2, $128.9,128.5,128.3,127.0,126.9,124.1,123.8,122.8,120.4,116.2$, 49.3, 45.1, 34.1, 31.5, 30.5, 26.2, 26.1, 24.9, 23.1, 22.9; HRMS-ESI: calcd for $[\mathrm{M}-\mathrm{H}]^{+}$622.7901, found 622.7902 .

4.1.2.22. $\mathrm{N}$-(9-(2-(4-Chlorophenyl)-4,5-diphenyl-1H-imidazol-1-yl) nonyl)- 1,2,3,4-tetrahydroacridin-9-amine (5). Intermediate 1d was treated with benzil, 4-chlorobenzaldehyde and ammonium acetate according to general procedure to give the desired product $\mathbf{5}$ as a yellow solid (43\% yield): m.p. $153-154{ }^{\circ} \mathrm{C}$; IR $(\mathrm{KBr}) \nu_{\max } / \mathrm{cm}^{-1}$ : 3379, 3053, 2931, 2854, 1495, 836, 770, 697; ${ }^{1} \mathrm{H} \mathrm{NMR}\left(\mathrm{CDCl}_{3}\right) \delta 7.93$ $(\mathrm{d}, 1 \mathrm{H}, J=8.4 \mathrm{~Hz}), 7.89(\mathrm{~d}, 1 \mathrm{H}, J=8.1 \mathrm{~Hz}), 7.63(\mathrm{~d}, 2 \mathrm{H}, J=8.7 \mathrm{~Hz})$,
7.58-7.28 (m, 11H), 7.24-7.09 (m, 3H), 4.00-3.78 (br, 1H), $3.85(\mathrm{t}$, $2 \mathrm{H}, J=7.5 \mathrm{~Hz}), 3.44(\mathrm{t}, 2 \mathrm{H}, J=7.2 \mathrm{~Hz}), 3.12-2.98(\mathrm{~m}, 2 \mathrm{H}), 2.76-2.63$ (m, 2H), 1.98-1.84 (m, 4H), 1.59 (qn, $2 \mathrm{H}, J=7.2 \mathrm{~Hz}), 1.38-1.20$ (m, $4 \mathrm{H}), 1.20-0.86(\mathrm{~m}, 8 \mathrm{H}) ;{ }^{13} \mathrm{C} \mathrm{NMR}\left(\mathrm{CDCl}_{3}\right) \delta 158.7,150.8,147.7,146.5$, $138.1,135.0,134.5,131.5,131.1,130.5,130.2,130.1,129.2,129.0,128.8$, $128.3,128.2,126.9,126.5,123.7,123.0,120.4,116.0,49.6,44.9,34.3$, 31.9, 30.4, 29.3, 29.2, 28.6, 27.0, 26.2, 24.9, 23.2, 23.0; HRMS-ESI: calcd for $[\mathrm{M}-\mathrm{H}]^{+}$654.3179, found 654.3195 .

4.1.2.23. $\mathrm{N}$-(10-(2-(4-Chlorophenyl)-4,5-diphenyl-1H-imidazol-1-yl) decyl)-1,2,3,4-tetrahydroacridin-9-amine (6). Intermediate $\mathbf{1 e}$ was treated with benzil, 4-chlorobenzaldehyde and ammonium acetate according to general procedure to give the desired product $\mathbf{6}$ as a yellow solid (33\% yield): m.p. $52-53{ }^{\circ} \mathrm{C}$; IR $(\mathrm{KBr}) \nu_{\max } / \mathrm{cm}^{-1}: 3422$, $3058,2925,2852,1499,834,760,696 ;{ }^{1} \mathrm{H} \mathrm{NMR}\left(\mathrm{CDCl}_{3}\right) \delta 7.94(\mathrm{~d}, 1 \mathrm{H}$, $J=8.7 \mathrm{~Hz}), 7.90(\mathrm{~d}, 1 \mathrm{H}, J=8.7 \mathrm{~Hz}), 7.63(\mathrm{~d}, 2 \mathrm{H}, J=8.4 \mathrm{~Hz}), 7.58-7.28$ $(\mathrm{m}, 11 \mathrm{H}), 7.24-7.09(\mathrm{~m}, 3 \mathrm{H}), 3.92(\mathrm{br}, 1 \mathrm{H}), 3.85(\mathrm{t}, 2 \mathrm{H}, J=7.6 \mathrm{~Hz})$, $3.44(\mathrm{t}, 2 \mathrm{H}, J=7.2 \mathrm{~Hz}), 3.14-2.98(\mathrm{~m}, 2 \mathrm{H}), 2.78-2.60(\mathrm{~m}, 2 \mathrm{H}), 2.00$ $1.84(\mathrm{~m}, 4 \mathrm{H}), 1.61$ (qn, $2 \mathrm{H}, J=7.2 \mathrm{~Hz}), 1.45-0.86(\mathrm{~m}, 14 \mathrm{H}) ;{ }^{13} \mathrm{C} \mathrm{NMR}$ $\left(\mathrm{CDCl}_{3}\right) \delta 158.6,150.9,147.7,146.5,138.1,134.9,134.5,131.5,131.1$, $130.5,130.2,130.1,129.2,129.0,128.9,128.8,128.3,128.2,126.9$, 126.5, 123.7, 123.0, 120.4, 116.0, 49.6, 44.9, 34.2, 31.9, 30.4, 29.4, 29.3, 29.2, 28.7, 27.0, 26.2, 24.9, 23.2, 23.0; HRMS-ESI: calcd for $[\mathrm{M}-\mathrm{H}]^{+} 668.3448$, found 668.3459 .

4.1.3. General procedure for the preparation of bis(n)-lophines $7 \boldsymbol{a}-\boldsymbol{e}$

A mixture of benzil $(1.0 \mathrm{mmol})$, diamine $(0.5 \mathrm{mmol})$, benzaldehyde $(1.0 \mathrm{mmol})$, ammonium acetate $(1.0 \mathrm{mmol})$ and $\mathrm{InCl}_{3}$ (10 $\mathrm{mol} \%$ ) in absolute ethanol $(1 \mathrm{~mL})$, placed in a round bottom flask fitted with a condenser and drying tube, was stirred under reflux for $24 \mathrm{~h}$. The solvent was removed under reduced pressure, and the crude product purified by column chromatography (eluting with hexane-ethyl acetate-triethylamine, 40:59:1) to give the desired product.

4.1.3.1. 2,4,5-Triphenyl-1-(10-(2,4,5-triphenyl-1H-imidazol-1-yl) decyl)-1H-imidazole (7a). Following the general procedure, 1,10decanodiamine, benzil, benzaldehyde and ammonium acetate afforded product $7 \mathbf{a}$ as a pale yellow solid (22\% yield): m.p. 165$166{ }^{\circ} \mathrm{C}$; IR $(\mathrm{KBr}) \nu_{\max } / \mathrm{cm}^{-1}$ : 3059, 2925, 2852, 1601, 1501, 1443, 773, 696; ${ }^{1} \mathrm{H}$ NMR $\left(\mathrm{CDCl}_{3}\right) \delta$ 7.71-7.64 (m, 4H), 7.56-7.37 (m, 20H), $7.23-7.09(\mathrm{~m}, 6 \mathrm{H}), 3.84(\mathrm{t}, 4 \mathrm{H}, J=7.6 \mathrm{~Hz}), 1.38-1.22(\mathrm{~m}, 4 \mathrm{H}), 0.96-$ $0.78(\mathrm{~m}, 12 \mathrm{H}) ;{ }^{13} \mathrm{C} \mathrm{NMR}\left(\mathrm{CDCl}_{3}\right) \delta 147.8,137.9,134.8,131.8,131.7$, 131.2, 129.8, 129.4, 129.2, 129.0, 128.8, 128.2, 127.0, 126.4, 44.9, 30.5, 29.0, 28.6, 26.2; HRMS-ESI: calcd for $[\mathrm{M}-\mathrm{H}]^{+} 732.0039$, found 732.0035 .

4.1.3.2. 2,4,5-Triphenyl-1-(9-(2,4,5-triphenyl-1H-imidazol-1-yl) nonyl)-1H-imidazole (7b). Following the general procedure, 1,9nonanodiamine, benzil, benzaldehyde and ammonium acetate afforded product $\mathbf{7 b}$ as a pale yellow solid (42\% yield): m.p. $83-$ $84{ }^{\circ} \mathrm{C}$; IR $(\mathrm{KBr}) \nu_{\max } / \mathrm{cm}^{-1}: 3057,2925,2852,1600,1499,1442,773$, $695 ;{ }^{1} \mathrm{H} \mathrm{NMR}\left(\mathrm{CDCl}_{3}\right) \delta 7.70-7.63(\mathrm{~m}, 4 \mathrm{H}), 7.56-7.34(\mathrm{~m}, 20 \mathrm{H}), 7.23-$ $7.08(\mathrm{~m}, 6 \mathrm{H}), 3.84(\mathrm{t}, 4 \mathrm{H}, J=7.3 \mathrm{~Hz}), 1.38-1.18(\mathrm{~m}, 4 \mathrm{H}), 0.92-0.68(\mathrm{~m}$, $10 \mathrm{H}) ;{ }^{13} \mathrm{C} \mathrm{NMR}\left(\mathrm{CDCl}_{3}\right) \delta 147.8,137.9,134.7,131.8,131.7,131.2,129.7$, 129.3, 129.2, 128.9, 128.7, 128.2, 127.0, 126.4, 44.8, 30.4, 28.7, 28.5, 26.2; HRMS-ESI: calcd for $[\mathrm{M}-\mathrm{H}]^{+}$717.9770, found 717.9766.

4.1.3.3. 2,4,5-Triphenyl-1-(8-(2,4,5-triphenyl-1H-imidazol-1-yl) octyl)-1H-imidazole (7c). Following the general procedure, 1,8octanodiamine, benzil, benzaldehyde and ammonium acetate afforded product 7c as a white solid (37\% yield): m.p. $150-151^{\circ} \mathrm{C}$; IR $(\mathrm{KBr})$ $\nu_{\max } / \mathrm{cm}^{-1}$ : 3056, 2933, 2856, 1600, 1479, 1442, 773, 696; ${ }^{1} \mathrm{H}$ NMR $\left(\mathrm{CDCl}_{3}\right) \delta 7.65(\mathrm{~d}, 4 \mathrm{H}, J=7.8 \mathrm{~Hz}), 7.52(\mathrm{~d}, 4 \mathrm{H}, J=7.8 \mathrm{~Hz}), 7.48-7.34(\mathrm{~m}$, $16 \mathrm{H}), 7.23-7.08(\mathrm{~m}, 6 \mathrm{H}), 3.81(\mathrm{t}, 4 \mathrm{H}, J=7.5 \mathrm{~Hz}), 1.21(\mathrm{qn}, 4 \mathrm{H}, J=7.5 \mathrm{~Hz})$, 
$0.81-0.60(\mathrm{~m}, 8 \mathrm{H}) ;{ }^{13} \mathrm{C}$ NMR $\left(\mathrm{CDCl}_{3}\right) \delta 147.8,137.8,134.7,131.7,131.6$, 131.1, 129.7,129.3,129.2,128.9,128.7,128.2,126.9,126.4, 44.7, 30.3, 28.2, 26.0; HRMS-ESI: calcd for $[\mathrm{M}-\mathrm{H}]^{+}$703.9501, found 703.9473.

4.1.3.4. 2,4,5-Triphenyl-1-(7-(2,4,5-triphenyl-1H-imidazol-1-yl)heptyl)-1H-imidazole (7d). Following the general procedure, 1,7heptanodiamine, benzil, benzaldehyde and ammonium acetate afforded product 7d as white solid (29\% yield): m.p. $84-85^{\circ} \mathrm{C}$; IR $(\mathrm{KBr})$ $\nu_{\max } / \mathrm{cm}^{-1}: 3058,2929,2856,1600,1500,1442,773,696 ;{ }^{1} \mathrm{H}$ NMR $\left(\mathrm{CDCl}_{3}\right) \delta 7.63(\mathrm{~d}, 4 \mathrm{H}, J=7.2 \mathrm{~Hz}), 7.54-7.47(\mathrm{~m}, 4 \mathrm{H}), 7.47-7.33(\mathrm{~m}, 16 \mathrm{H})$, $7.23-7.08(\mathrm{~m}, 6 \mathrm{H}), 3.77(\mathrm{t}, 4 \mathrm{H}, J=7.5 \mathrm{~Hz}), 1.20-1.08(\mathrm{~m}, 4 \mathrm{H}), 0.74-0.50$ $(\mathrm{m}, 6 \mathrm{H}) ;{ }^{13} \mathrm{C} \mathrm{NMR}\left(\mathrm{CDCl}_{3}\right) \delta 147.7,137.8,134.7,131.7,131.6,131.1,129.7$, 129.3, 129.2, 129.0, 128.7, 128.2, 126.9, 126.4, 44.6, 30.2, 27.7, 25.9; HRMS-ESI: calcd for $[\mathrm{M}-\mathrm{H}]^{+} 689.9233$, found 689.9232 .

4.1.3.5. 2,4,5-Triphenyl-1-(6-(2,4,5-triphenyl-1H-imidazol-1-yl)hex$y l)$-1H-imidazole $(\mathbf{7} \boldsymbol{e})$. Following the general procedure, 1,6hexanodiamine, benzil, benzaldehyde and ammonium acetate afforded product 7e as a white solid (30\% yield): m.p. $166-167{ }^{\circ} \mathrm{C}$; IR $(K B r) \nu_{\max } / \mathrm{cm}^{-1}: 3060,2937,2858,1600,1500,1442,773,698 ;{ }^{1} \mathrm{H}$ NMR $\left(\mathrm{CDCl}_{3}\right) \delta 7.63-7.57(\mathrm{~m}, 4 \mathrm{H}), 7.54-7.48(\mathrm{~m}, 4 \mathrm{H}), 7.45-7.29(\mathrm{~m}$, $16 \mathrm{H}), 7.23-7.08(\mathrm{~m}, 6 \mathrm{H}), 3.71(\mathrm{t}, 4 \mathrm{H}, J=7.5 \mathrm{~Hz}), 1.14-0.98(\mathrm{~m}, 4 \mathrm{H})$, $0.64-0.48(\mathrm{~m}, 4 \mathrm{H}) ;{ }^{13} \mathrm{C}$ NMR $\left(\mathrm{CDCl}_{3}\right) \delta 147.7,137.8,134.6,131.6$, 131.5, 131.0, 129.6, 129.2, 129.1, 128.9, 128.7, 128.2, 126.9, 126.4, 44.5, 30.0, 25.3; HRMS-ESI: calcd for $[\mathrm{M}-\mathrm{H}]^{+} 675.8964$, found 675.8976 .

\subsection{In vitro assay of brain $A C h E$ and BuChE activities}

Acetylcholinesterase (AChE) and butyrylcholinesterase (BuChE) activities were measured by the colorimetric method of Ellman et al. [34] with some minor modifications, adapted for determining the effects of analogs on enzyme activity in supernatants of rat's brain homogenates and human plasma, respectively. Adults Wistar male rats (2-3 months) were supplied by Department of BiochemistryUFRGS. Animals were housed in plastic cages and maintained at $22-23{ }^{\circ} \mathrm{C}$ under a $12 \mathrm{~h}$ light/dark cycle (lights on at 7:00 a.m.) with free access to food and water. All experiments were following and approved by the Animal Care and Ethics Committee of the Universidade Federal do Rio Grande do Sul. For AChE sources, rats were decapitated, the brain was rapidly dissected on ice into cortex and homogenized in cold $10 \mathrm{mM}$ Tris- $\mathrm{HCl}$ buffer, pH 7.2 containing $160 \mathrm{mM}$ sucrose. The homogenates were centrifugated at $10,000 \times \mathrm{g}$ for $10 \mathrm{~min}$ at $4{ }^{\circ} \mathrm{C}$; supernatants were used as AChE sources, kept into aliquots and stored at $-20^{\circ} \mathrm{C}$. For BuChE sources, human plasma was obtained from 5 healthy volunteers (24-60 years old) of both sexes with written consent and the protocol was approved by the Human Ethic Committee of the Universidade Federal do Rio Grande do Sul. The samples were centrifugated at $1000 \times \mathrm{g}$ for $15 \mathrm{~min}$ at room temperature and aliquots were stored at $-20^{\circ} \mathrm{C}$.

AChE and BuChE enzyme samples, in $20 \mathrm{mM}$ phosphate buffer $\mathrm{pH}$ 7.4 , were incubated $150 \mathrm{~s}$ with acetylthiocholine iodide or butyrylthiocholine iodide $(0.8 \mathrm{mM})$, respectively, in the presence of $10 \mathrm{mM} 5,5^{\prime}$ dithiobis-(2-nitrobenzoic acid) (DTNB), for color development (all chemicals from Sigma). Production of the yellow anion of 5-thio-2nitrobenzoic acid was measured with a SPECTRAmax 190, 96-well plate reader, at $415 \mathrm{~nm}$. Protein concentration for both type of samples were measured by determined by Peterson's modification of the procedure of Lowry et al. [35], using bovine serum albumin as standard.

\subsection{Statistical analysis}

The $\mathrm{IC}_{50}$ values were determined from a plot of activity value of $\log$ (inhibitor) vs. response, which was processed by a software of GraFit 6.0. All results are calculated as mean \pm SD of five independent measurements, each performed in triplicate.

\section{Acknowledgements}

The authors wish to thank the following Brazilian agencies for financial support and fellowships: CNPq, FAPERGS, PROPESQ - UFRGS.

\section{Appendix A. Supplementary data}

Supplementary data related to this article can be found at http:// dx.doi.org/10.1016/j.ejmech.2013.01.029.

\section{References}

[1] M. Goedert, M.G. Spillantini, Science 314 (2006) 777-781.

[2] A.V. Terry Jr., J.J. Buccafusco, J. Pharmacol. Exp. Ther. 306 (2003) 821-827.

[3] R.T. Bartus, Exp. Neurol. 163 (2000) 495-529.

[4] S. Rizzo, A. Bisi, M. Bartolini, F. Mancini, F. Belluti, S. Gobbi, V. Andrisano, A. Rampa, Eur. J. Med. Chem. 46 (2011) 4336-4343.

[5] M. Kozurkova, S. Hamulakova, Z. Gazova, H. Paulikova, P. Kristian, Pharmaceuticals 4 (2011) 382-418.

[6] J.J. Bornstein, T.J. Eckroat, J.L. Houghton, C.K. Jones, K.D. Green, S. GarneauTsodikova, Med. Chem. Commun. 2 (2011) 406-412

[7] C. Ronco, L. Jean, H. Outaabout, P.Y. Renard, Eur. J. Org. Chem. (2011) 302-310.

[8] R.T. Delfino, T.S. Ribeiro, J.D. Figueroa-Villar, J. Braz. Chem. Soc. 20 (2009) 407-428.

[9] D.A. Smith, Am. J. Health-syst. Pharm. 66 (2009) 899-907.

[10] M.I. Fernández-Bachiller, C. Pérez, L. Monjas, J. Rademann, M.I. RodríguezFranco, J. Med. Chem. 55 (2012) 1303-1317.

[11] G.T. Grossberg, V. Pejovic, M.L. Miller, S.M. Graham, Dement. Geriatr. Cogn. Disord. 27 (2009) 164-172.

[12] E.K. Perry, R.H. Perry, G. Blessed, B.E. Tomlinson, Neuropathol. Appl. Neurobiol. 4 (1978) 273-277.

[13] J.L. Sussman, M. Harel, F. Frolow, C. Oefner, A. Goldman, L. Toker, I. Silman, Science 253 (1991) 872-879.

[14] Y. Nicolet, O. Lockridge, P. Masson, J.C. Fontecilla-Camps, F. Nachon, J. Biol. Chem. 278 (2003) 41141-41147.

[15] Y.P. Pang, P. Quiram, T. Jelacic, F. Hong, S. Brimijoin, J. Biol. Chem. 271 (1996) 23646-23649.

[16] P. Camps, X. Formosa, C. Galdeano, T. Gómez, D. Muñoz-Torrero M. Scarpellini, E. Viayna, A. Badia, M.V. Clos, A. Camins, M. Pallàs, M. Bartolini, F. Mancini, V. Andrisano, J. Estelrich, M. Lizondo, A. Bidon-Chanal, F.J. Luque, J. Med. Chem. 51 (2008) 3588-3598.

[17] A. Badia, J.E. Baños, P. Camps, J. Contreras, D.M. Görbig, D. Muñoz-Torrero, M. Simón, N.M. Vivas, Bioorg. Med. Chem. 6 (1998) 427-440.

[18] P. Camps, X. Formosa, C. Galdeano, T. Gómez, D. Muñoz-Torrero, L. Ramírez, E. Viayna, E. Gómez, N. Isambert, R. Lavilla, A. Badia, M.V. Clos, M. Bartolini, F. Mancini, V. Andrisano, A. Bidon-Chanal, O. Huertas, T. Dafni, F.J. Luque, Chem. Biol. Interact. 187 (2010) 411-415.

[19] P. Camps, X. Formosa, C. Galdeano, D. Muñoz-Torrero, L. Ramírez, E. Gómez, N. Isambert, R. Lavilla, A. Badia, M.V. Clos, M. Bartolini, F. Mancini, V. Andrisano, M.P. Arce, M.I. Rodríguez-Franco, O. Huertas, T. Dafni, F.J. Luque, J. Med. Chem. 52 (2009) 5365-5379.

[20] D. Shao, C.Zou, C. Luo, X. Tang, Y. Li, Bioorg. Med. Chem. Lett.14(2004)4639-4642.

[21] D. Alonso, I. Dorronsoro, L. Rubio, P. Muñoz, E. García-Palomero, M. Del Monte, A. Bidon-Chanal, M. Orozco, F.J. Luque, A. Castro, M. Medina, A. Martínez, Bioorg. Med. Chem. 13 (2005) 6588-6597.

[22] P. Muñoz-Ruiz, L. Rubio, E. García-Palomero, I. Dorronsoro, M. del MonteMillán, R. Valenzuela, P. Usán, C. de Austria, M. Bartolini, V. Andrisano, A. Bidon-Chanal, M. Orozco, F.J. Luque, M. Medina, A. Martínez, J. Med. Chem. 48 (2005) 7223-7233.

[23] D. Antequera, M. Bolos, C. Spuch, C. Pascual, I. Ferrer, M.I. Fernandez-Bachiller, M.I. Rodríguez-Franco, E. Carro, Neurobiol. Dis. 46 (2012) 682-691.

[24] D.S. Pisoni, J.S. da Costa, D. Gamba, C.L. Petzhold, A.C.A. Borges, M.A. Ceschi, P. Lunardi, C.A.S. Gonçalves, Eur. J. Med. Chem. 45 (2010) 526-535.

[25] J.S. da Costa, D.S. Pisoni, C.B. da Silva, C.L. Petzhold, D. Russowsky, M.A. Ceschi, J. Braz. Chem. Soc. 20 (2009) 1448-1454.

[26] M.V. Marques, M.M. Ruthner, L.A.M. Fontoura, D. Russowsky, J. Braz. Chem. Soc. 23 (2012) 171-179.

[27] C.S. Schwalm, M.A. Ceschi, D. Russowsky, J. Braz. Chem. Soc. 22 (2011) 623-636.

[28] S. Samai, G.C. Nandi, P. Singh, M.S. Singh, Tetrahedron 65 (2009) 10155-10161.

[29] S. Das Sharma, P. Hazarika, D. Konwar, Tetrahedron Lett. 49 (2008) 2216-2220.

[30] M.K. Hu, C.F. Lu, Tetrahedron Lett. 41 (2000) 1815-1818

[31] M.K. Hu, J. Pharm. Pharmacol. 53 (2001) 83-88.

[32] W. Luo, Y.P. Li, Y. He, S.L. Huang, J.H. Tan, T.M. Ou, D. Li, L.Q. Gu, Z.S. Huang Bioorg. Med. Chem. 19 (2011) 763-770.

[33] D. Yanover, M. Kaftory, Acta Cryst. E65 (2009) o711.

[34] G.L. Ellman, K.D. Courtney, V. Andres, R.M. Featherstone, Biochem. Pharmacol. 7 (1961) 88-95.

[35] G.L. Peterson, A simplification of the protein assay method of Lowry et al. which is more generally applicable, Anal. Biochem. 83 (1977) 346-356. 